\title{
Unicast and Multicast QoS Routing with Soft Constraint Logic Programming
}

\author{
STEFANO BISTARELLI \\ Università Chieti-Pescara, Istituto di Informatica e Telematica \\ UGO MONTANARI \\ Università di Pisa \\ FRANCESCA ROSSI \\ Università di Padova \\ and \\ FRANCESCO SANTINI \\ IMT - Institute for Advanced Studies, Istituto di Informatica e Telematica
}

\begin{abstract}
We present a formal model to represent and solve the unicast/multicast routing problem in networks with Quality of Service (QoS) requirements. To attain this, first we translate the network adapting it to a weighted graph (unicast) or and-or graph (multicast), where the weight on a connector corresponds to the multidimensional cost of sending a packet on the related network link: each component of the weights vector represents a different QoS metric value (e.g. bandwidth, cost, delay, packet loss). The second step consists in writing this graph as a program in Soft Constraint Logic Programming (SCLP): the engine of this framework is then able to find the best paths/trees by optimizing their costs and solving the constraints imposed on them (e.g. delay $\leq 40 \mathrm{msec}$ ), thus finding a solution to QoS routing problems. Moreover, c-semiring structures are a convenient tool to model QoS metrics. At last, we provide an implementation of the framework over scale-free networks and we suggest how the performance can be improved.

Categories and Subject Descriptors: D.3.2 [Programming Languages]: Language Classifications - Constraint and logic languages; D.3.3 [Programming Languages]: Language Constructs and Features-Constraints; C.2.3 [Computer-Communication Networks]: Network Operations-Network management; F.4.1 [Mathematical Logic And Formal Languages]: Mathematical Logic - Logic and constraint programming
\end{abstract}

General Terms: Languages, Measurement, Theory

Author's address: Stefano Bistarelli, Dipartimento di Scienze, Università di Chieti-Pescara, Viale Pindaro 42, Pescara, 65127, Italy. bista@sci.unich.it. - Institute for Informatics and Telematics, Via G. Moruzzi 1, 56100 Pisa, Italy. stefano.bistarelli@iit.cnr.it.

Ugo Montanari, Dipartimento di Informatica, Università di Pisa, Largo Bruno Pontecorvo 3, Pisa, 56127, Italy. ugo@di.unipi.it

Francesca Rossi, Dipartimento di Matematica Pura e Applicata, Università di Padova, Via Trieste 6335121 Padova, Italy. frossi@math.unipd.it

Francesco Santini, IMT - Institute for Advanced Studies, Piazza San Ponziano 6, 55100 Lucca, Italy. f.santini@imtlucca.it. - Institute for Informatics and Telematics, Via G. Moruzzi 1, 56100 Pisa, Italy. francesco.santini@iit.cnr.it.

Permission to make digital/hard copy of all or part of this material without fee for personal or classroom use provided that the copies are not made or distributed for profit or commercial advantage, the ACM copyright/server notice, the title of the publication, and its date appear, and notice is given that copying is by permission of the ACM, Inc. To copy otherwise, to republish, to post on servers, or to redistribute to lists requires prior specific permission and/or a fee.

(C) 20YY ACM 0000-0000/YY/00-0001 $\$ 5.00$ 


\section{INTRODUCTION}

Towards the second half of the nineties, Internet Engineering Task Force (IETF) and the research community have proposed many service models and mechanisms [Xiao and Ni 1999; Paul and Raghavan 2002] to meet the demand for network Quality of Service (QoS). The reason is that traditional networks cannot recognize a priority associated with data, because they handle network traffic with the best effort principles. According to this treatment, the network does not provide any guarantees that data is delivered or that a user is assisted with a guaranteed QoS level or a certain priority (due to congestions). In best effort networks, all users obtain exactly the same treatment. However nowadays, networked applications, such as Enterprise Resource Planning (ERP), data mining, distance learning, resource discovery, e-commerce, and distribution of multimedia-content, stock quotes, and news, are bandwidth hungry, need a certain "timeliness" (i.e. events occurring at a suitable and opportune time) and are also mission critical.

For all these reasons, the routing problem has naturally been extended to include and to guarantee QoS requirements [Younis and Fahmy 2003; Xiao and Ni 1999; Paul and Raghavan 2002], and consequently is usually abbreviated to QoS routing. As defined in [Crawley et al. 1998], QoS is "a set of service requirements to be met by the network while transporting a flow", where a flow is "a packet stream from source to a destination (unicast or multicast) with an associated Quality of Service (QoS)". To be implemented and subsequently satisfied, service requirements have to be expressed in some measurable QoS metrics, such as bandwidth, number of hops, delay, jitter, cost and loss probability of packets.

This paper combines and extends the two works presented in [Bistarelli et al. 2002] and [Bistarelli et al. 2007]. First, we detail the modelling procedure to represent and solve plain Shortest Path (SP) [Cormen et al. 1990] problems with Soft Constraint Logic Programming (see Sec. 2). We consider several versions of SP problems, from the classical one to the multi-criteria case (i.e. many costs to be optimized), from partially-ordered problems to those that are based on modalities associated to the use of the arcs (i.e. modality-based), and we show how to model and solve them via SCLP programs. The basic idea is that the paths represent network routes, edge costs represent QoS metric values, and our aim is to guarantee the requested QoS on the found unicast routes, by satisfying the QoS constraints and optimizing the cost of the route at the same time. The different criteria can be, for example, maximizing the global bandwidth and minimizing the delay that can be experienced on a end-to-end communication.

Then, extending the unicast solution, we suggest a formal model to represent and solve the multicast routing problem in multicast networks (i.e. networks supporting the multicast delivery schema) that need QoS support. To attain this, we draw the network adapting it to a weighted and-or graph [Martelli and Montanari 1978], where the weight on a connector corresponds to the cost of sending a packet on the network link modelled by that connector. Then, we translate the hypergraph in a SCLP program and we show how the semantic of this program computes the best tree in the corresponding and-or graph. We apply this result to find, from a given source node in the network, the multicast distribution tree having the minimum cost and reaching all the destination nodes of the multicast communication. The

ACM Journal Name, Vol. V, No. N, $20 Y$ Y. 
costs of the connectors can be described as vectors (multidimensional costs), each component representing a different QoS metric value. We show also how modalities can be added to multicast problems, and how the computational complexity of this framework can be reduced. Therefore, in this paper we present a complete formal model to represent and solve the unicast/multicast QoS routing problem.

SCLP programs are logic programs where each ground atom can be seen as an instantiated soft constraint [Bistarelli et al. 1995; 1997b] and it can be associated with an element taken from a set. Formally, this set is a $c$-semiring [Bistarelli 2004] (or simply semiring in the following), that is, a set plus two operations, + and $\times$, which basically say how to combine constraints and how to compare them. The presence of these two operations allows to replace the usual boolean algebra for logic programming with a more general algebra where logical and and logical or are replaced by the two semiring operations. In this way, the underlying logic programming engine provides a natural tool to specify and solve combinatorial problems, while the soft constraint machinery provides greater expressivity and flexibility.

The most important features of the adopted framework are: first, is that SCLP is a declarative programming environment and, thus, is relatively easy to specify a lot of different problems, ranging from paths to trees. The model can be used to easily specify the problem, which can be then translated and solved with a fast solver; however, our goal is to improve the performance also for our implementation. The second reason is that the semiring structure is a very flexible and parametric tool where to represent several and different cost models, with respect to QoS metrics; obviously, the same SCLP programming environment and operational semantic engine can be used with all these different semirings. Finally, since QoS routing problem can be in general NP-Complete, SCLP promises to be suitable tool, due to its ability for solving combinatorial problems (as shown in [Georget and Codognet 1998]).

\subsection{Related Works}

Concerning the related works, in [de Nicola et al. 2003] and [Hirsch and Tuosto 2005] the authors adopt a hypergraph model in joint with semirings too, but the minimal path between two nodes (thus, not over an entire tree) is computed via a graphical calculous instead of SCLP. At the moment, all these frameworks are not comparable from the computational performance point of view, since they have not yet been implemented. Even the work in [Mammeri 2004] presents some general algebraic operators in order to handle QoS in networks, but without any practical results. We compare our work only with other theoretical frameworks, since our study aims at representing general routing constraints in order to solve different problems: due to the complexity of QoS routing, state-of-the-art practical solutions (presented in Sec. 3.2 and Sec. 3.3) deal only with a subset of metrics and constraints. On the other hand, a more general framework can help to analyze the problem from a global point of view, not linked to specific algorithms. With Declarative routing [Loo et al. 2005], a routing protocol is implemented by writing a simple query in a declarative query language (like Datalog as in [Loo et al. 2005]), which is then executed in a distributed fashion at some or all of the nodes. It is based on the observation that recursive query languages are a natural-fit for expressing routing 
protocols. However, the authors of [Loo et al. 2005] did not go deep in modelling QoS features, and we think that c-semirings represent a very good method to include these metrics.

To go further, aside the elegant formalization due to the SCLP framework, we build a bridge to a real implementation of the model (Sec. 7.2) and several ideas to improve the experienced performance. The final tool can be used to quickly prototype and test different routing paths. Therefore, our paper vertically cover the problem: from theoretical to practical aspects, without reaching the performance of existing routing algorithms implemented inside the routers, but thoroughly and expressively facing the problem. As far as we know, other formal representations completely miss this practical implementation.

\subsection{Structure of the paper}

The remainder of this paper is organized as follows. In Sec. 2 we describe the SCLP framework, while in Sec. 3 we complete the background by introducing the multicast/unicast QoS routing: we show that the problem of defining a route that has to be optimized and is subject to constraints concerning QoS metrics, is, in general, a NP-Complete problem. Then, we report some of the solutions, mostly through heuristics, given in the real world. Section 4 proposes how to model and solve the unicast QoS routing with SCLP, considering also problems with multidimensional costs (i.e. multi-criteria problems) and based on modalities of use associated with the links of the network: for example, if we need to find a route by using only wireless, and/or wired and/or encrypted links (i.e. modality-based problems). Section 5 outlines a similar framework, based on hypergraph and SCLP, for the management of the multicast QoS routing: we show how to translate a network in a corresponding and-or graph and then we compute the best distribution tree by using SCLP. Even in this case we extend the model to include problems with modalities. Section 6 gives some important considerations about semirings that improve the model when the costs of the network links are multidimensional and partially ordered: this is the common case, since an effective measurement of QoS will necessarily involve a collection of measures. We show also how we can limit the number of partially ordered solutions with ad-hoc semirings, which apply a total order on the tuples of cost values by following a set of weights defined to satisfy the user. Section 7 presents a practical implementation of the model by solving the problem over scale-free [Barabasi and Albert 1999] networks, which properly model the topology of Internet. This implementation has been developed to demonstrate that performance improvements are necessary. These improvements can be achieved with the mechanisms explained in Sec. 8, as tabling and branch-and-bound (as our implementation in ECLiPSe [Apt and Wallace 2007] shows). At last, Sec. 9 ends the paper with the final conclusions and ideas about future work.

\section{SOFT CONSTRAINT LOGIC PROGRAMMING}

The SCLP framework [Bistarelli 2004; Bistarelli et al. 1997a; Georget and Codognet 1998], is based on the notion of c-semiring introduced in [Bistarelli et al. 1995; 1997b] (c-semiring and semiring terms will be used as synonyms in this paper). A semiring $S$ is a tuple $\langle A,+, \times, \mathbf{0}, \mathbf{1}\rangle$ where $A$ is a set with two special elements $(\mathbf{0}, \mathbf{1} \in A)$ and with two operations + and $\times$ that satisfy certain properties: + 
is defined over (possibly infinite) sets of elements of $A$ and thus is commutative, associative, idempotent, it is closed and $\mathbf{0}$ is its unit element and $\mathbf{1}$ is its absorbing element; $\times$ is closed, associative, commutative, distributes over,$+ \mathbf{1}$ is its unit element, and $\mathbf{0}$ is its absorbing element (for the exhaustive definition, please refer to [Bistarelli et al. 1997b]).

The + operation defines a partial order $\leq_{S}$ over $A$ such that $a \leq_{S} b$ iff $a+b=b$; we say that $a \leq_{S} b$ if $b$ represents a value better than $a$. Other properties related to the two operations are that + and $\times$ are monotone on $\leq_{S}, \mathbf{0}$ is its minimum and 1 its maximum, $\left\langle A, \leq_{S}\right\rangle$ is a complete lattice and + is its lub. Finally, if $\times$ is idempotent, then + distributes over $\times,\left\langle A, \leq_{S}\right\rangle$ is a complete distributive lattice and $\times$ its glb.

Semiring-based Constraint Satisfaction Problems (SCSPs) [Bistarelli 2004] are constraint problems where each variable instantiation is associated to an element of a c-semiring $A$ (to be interpreted as a cost, level of preference, ...), and constraints are combined via the $\times$ operation and compared via the $\leq_{S}$ ordering. Varying the set $A$ and the meaning of the + and $\times$ operations, we can represent many different kinds of problems, having features like fuzziness, probability, and optimization. Notice also that the cartesian product of two c-semirings is a c-semiring [Bistarelli et al. 1997b], and this can be fruitfully used to describe multi-criteria constraint satisfaction and optimization problems.

Constraint Logic Programming (CLP) [Jaffar and Maher 1994] extends Logic Programming by replacing term equalities with constraints and unification with constraint solving. The SCLP framework extends the classical CLP formalism in order to be able to handle also SCSP [Bistarelli et al. 1995; 1997b] problems. In passing from CLP to SCLP languages, we replace classical constraints with the more general SCSP constraints where we are able to assign a level of preference to each instantiated constraint (i.e. a ground atom). To do this, we also modify the notions of interpretation, model, model intersection, and others, since we have to take into account the semiring operations and not the usual CLP operations.

The fact that we have to combine several refutation paths when we have a partial order among the elements of the semiring (instead of a total one), can be fruitfully used in the context of this paper when we have an graph/hypergraph problems with incomparable costs associated to the edges/connectors. In fact, in the case of a partial order, the solution of the problem of finding the best path/tree should consist of all those paths/trees whose cost is not "dominated" by others.

Table I. A simple example of an SCLP program.

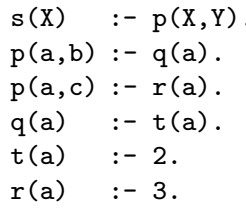

A simple example of an SCLP program over the semiring $\langle\mathbb{N}, \min ,+,+\infty, 0\rangle$, where $\mathbb{N}$ is the set of non-negative integers and $D=\{a, b, c\}$, is represented in 
Table I. The choice of this semiring allows us to represent constraint optimization problems where the semiring elements are the costs for the instantiated atoms. To better understand this Table, we briefly recall the SCLP syntax: a program is a set of clauses and each clause is composed by a head and a body. The head is just an atom, and the body is either a collection of atoms, or a value of the semiring, or a special symbol $(\square)$ to denote that it is empty. Clauses where the body is empty or it is just a semiring element are called facts and define predicates which represent constraints. When the body is empty, we interpret it as having the best semiring element (that is, 1).

The intuitive meaning of a semiring value like 3 associated to the atom $r(a)$ (in Table I) is that $r(a)$ costs 3 units. Thus the set $\mathbb{N}$ contains all possible costs, and the choice of the two operations min and + implies that we intend to minimize the sum of the costs. This gives us the possibility to select the atom instantiation which gives the minimum cost overall. Given a goal like $s(x)$ to this program, the operational semantics collects both a substitution for $x$ (in this case, $x=a$ ) and also a semiring value (in this case, 2) which represents the minimum cost among the costs for all derivations for $s(x)$. To find one of these solutions, it starts from the goal and uses the clauses as usual in logic programming, except that at each step two items are accumulated and combined with the current state: a substitution and a semiring value (both provided by the used clause). The combination of these two items with what is contained in the current goal is done via the usual combination of substitutions (for the substitution part) and via the multiplicative operation of the semiring (for the semiring value part), which in this example is + . Thus, in the example of goal $s(X)$, we get two possible solutions, both with substitution $X=a$ but with two different semiring values: 2 and 3 . Then, the combination of such two solutions via the min operation give us the semiring value 2 .

To extend the representation we briefly introduce semiring valuations [Wilson 2004], which are constraint satisfaction problems taking values in a commutative semiring, where the ordering is the transitive relation $a \leq b$ iff $\exists . a+c=b$. The lack of idempotency for the sum operator results in a weaker structure than absorptive semirings, that has proved useful whenever counting the number of solutions is of interest. Even if throughout the paper we will use the semiring definition given in [Bistarelli et al. 1995; 1997b] (i.e. with an idempotent + ), semiring valuations can be used for those metrics that need to be aggregated together from different solutions, e.g. the packet loss probability between two nodes $p$ and $v$ is computed with the probabilities of all the possible different paths connecting $p$ and $v$ in the graph. However, the associated reflexive and transitive relation $\leq$ satisfies relatively few properties, since adding constraints does not lead to worsen the solution, thus resulting in a non-monotonic framework [Wilson 2004].

\section{QOS ROUTING}

With Constraint-Based Routing (CBR) we refer to a class of routing algorithms that base path selection decisions on a set of requirements or constraints, in addition to destination criteria. These constraints may be imposed by administrative policies (i.e. policy routing), or by QoS requirements (i.e. QoS routing, as already cited in Sec. 1), and so they can be classified in two classes with different charac-

ACM Journal Name, Vol. V, No. N, 20 YY. 
teristics. The aim of CBR is to reduce the manual configuration and intervention required for attaining traffic engineering objectives [Rosen et al. 2001]; for this reason, CBR enhances the classical routing paradigm with special properties, such as being resource reservation-aware and demand-driven.

The routing associated with administration decisions is referred to as policy routing (or policy-based routing), and it is used to select paths that conform to administrative rules and Service Level Agreements (SLAs) stipulated among service providers and clients. In this way, routing decisions can be based not only on the destination location, but also on other factors such as applications or protocols used, size of packets, identity of the communicating entities, or in general, business related decisions. Policy constraints can help improving the global security of the network: constraints can be used to guarantee agreed service provisioning and safety from malicious users attempting to steal the resources not included in their contracts.

QoS routing attempts to simultaneously satisfy multiple QoS requirements requested by real-time applications: the requirements are usually expressed using metrics as, e.g. delay and bandwidth. Policy routing (or policy-based routing) is instead used to select paths that conform to imposed administrative rules. In this way, routing decisions can be based not only on the destination location, but also on factors such as used applications and protocols, size of packets, or identity of both source and destination end systems of the flow. Policy constraints can improve the global security of network infrastructure and are able to realize business related decisions.

Traditionally, QoS metrics can be organized into three distinct classes, depending on how they are combined along a path: they can be i) additive, ii) multiplicative or iii) concave [Wang and Crowcroft 1996]. They are defined as follows: with $n_{1}, n_{2}, n_{3} \ldots, n_{i}, n_{j}$ representing network nodes, let $m\left(n_{1}, n_{2}\right)$ be a metric value for the link connecting $n_{1}$ and $\left.n_{2}\right)$. For any path $P=\left(n, n_{2}, \ldots, n_{i}, n_{j}\right)$, the metric corresponding is:

-Additive, if $m(P)=m\left(n_{1}, n_{2}\right)+m\left(n_{2}, n_{3}\right)+\ldots+m\left(n_{i}, n_{j}\right)$ The additive metric of a path is the sum of the metric for all the links constituting the path. Some examples are delay, jitter (the delay variation on a network path), cost and hopcount.

- Multiplicative, if $m(P)=m\left(n_{1}, n_{2}\right) \times m\left(n_{2}, n_{3}\right) \cdots \times m\left(n_{i}, n_{j}\right)$ Multiplicative metric (equivalent to the additive one just by taking the logarithm of all costs) of a path consists in the multiplication of the metric values for all the links constituting the path. Example is reliability or loss probability.

- Concave, if $m(P)=\max / \min \left\{m\left(n_{1}, n_{2}\right), m\left(n_{2}, n_{3}\right), \ldots, m\left(n_{i}, n_{j}\right)\right\}$. The concave metric of a path is the maximum or the minimum of the metric values over all the links in the path. The classical example is bandwidth, meaning that the bandwidth of a path is determined by the link with the minimum available bandwidth, i.e. the bottleneck of the path. Other concave metrics can be represented for example by packet buffers or CPU usage of the routers along the path, or, however, something to be maximized and depending on the "weakest link".

Even if usually the metric classes are introduced for paths, most of times they can be suitable also for trees: consider, for example, if we need to find a global cost 
of the tree by summing up all the weights on the tree edges (i.e. additive), or if we want to maximize the bandwidth of bottleneck link (i.e. concave).

Given a node generating packets, we can classify network data delivery schemas into three main classes: i) unicast, when data is delivered from one sender to one specific recipient, providing one-to-one delivery, ii) broadcast, when data is instead delivered to all hosts, providing one-to-all delivery, and finally, iii) multicast, when data is delivered to all the selected hosts that have expressed interest; thus, this last method provides one-to-many delivery. We will concentrate on i) and iii).

\subsection{Two NP-Complete Problems}

When we use multiple QoS metrics, a typical scenario involves resources that are independent and allowed to take real or unbounded integer values [Kompella and Awduche 2001]. For example, it could be necessary to find a route with the objective of cost minimization (i.e. a quantitative constraint, optimizing a metric) and subject to a path delay $\leq 40 \mathrm{msec}$ (i.e. a boolean constraint, saying whether or not a route is feasible) at the same time, therefore we would have the set of constraints $C=($ delay $\leq 40, \min ($ cost $))$. In such scenarios, satisfying two boolean constraints, or a boolean constraint and a quantitative (optimization) constraint is NP-Complete [Younis and Fahmy 2003]. If all resources except one take bounded integer values, or if resources are dependent, then the problems can be solved in polynomial time [Chen and Nahrstedt 1998]. Most of the proposed algorithms in this area apply heuristics to reduce the complexity, as we will see in Sec. 3.2 and 3.3.

Unicast and multicast QoS routing can be reduced to two well-known and more general problems: respectively, Multi-Constrained Path (MCP) [Korkmaz and Krunz 2001; Paul and Raghavan 2002] and Steiner Tree (ST) [Winter 1987; Paul and Raghavan 2002] problems.In MCP, the problem is to find a path from node $s$ to node $t$ in a graph where each link is associated with $k$ non-negative additive weights, while satisfying a set of constraints $C$ on these weights.

There may be multiple different paths in the graph $G(N, E)$ that satisfy the same set of constraints. Such paths are said to be feasible. However, often it might be desirable to retrieve an optimal path, according to some criteria, and respecting also the bounds imposed by the constraints. This more difficult problem is known as the Multi-Constrained Optimal Path (MCOP) problem. Clearly, since the paths must be optimized according to some costs criteria, MCOP intersects the Shortest Path problem.

The MCP problem is a NP-Complete problem. The authors of [Garey and Johnson 1979] were the first to list the MCP problem with a number of metrics $m=2$ as being NP-complete, but they did not provide a proof. Wang and Crowcroft have provided this proof for $m \geq 2$ in [Wang and Crowcroft 1996] and [Wang 1999], which basically consisted of reducing the MCP problem for $m=2$ to an instance of the partition problem, a well-known NP-complete problem [Garey and Johnson 1979]. However, simulations performed in (for example) [Mieghem et al. 2001; Kuipers et al. 2004; Younis and Fahmy 2003] show that QoS routing may be practically tractable in some of the possible cases.

In the ST problem, given a set $S$ of vertices in a graph $G=(V, E)$, a solution interconnects them by a graph of minimum weight, where the weight is the sum of the weights of all edges. If $S=V$, the ST problem reduces to the Minimum 


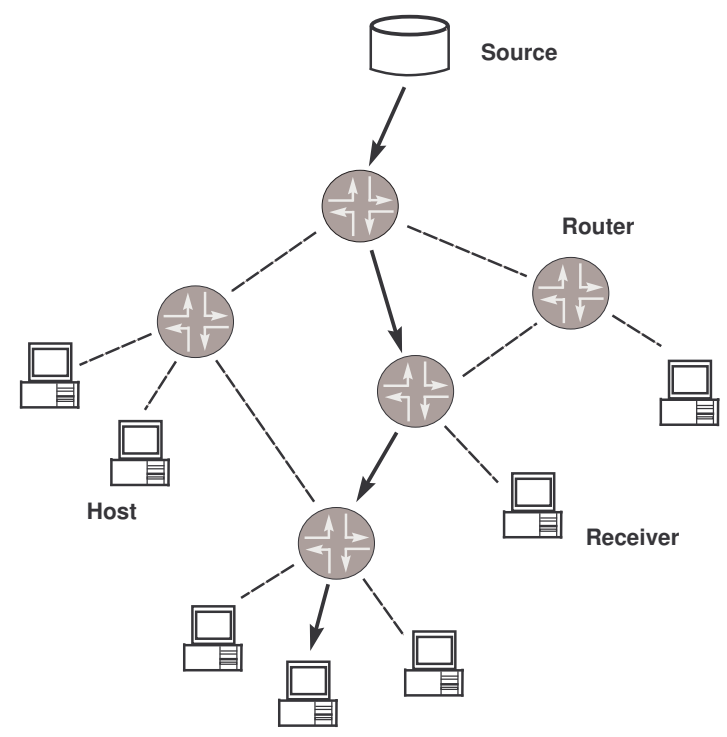

Fig. 1. An example of a unicast distribution between the source and the receiver. Oriented arcs highlight the path, while dashed lines correspond to links not traversed by the flow.

Spanning Tree (MST) problem[Cormen et al. 1990]. ST has been extended to Constrained Steiner Tree (CST), to include constraints concerning the weights of the links; for example, if we want that the sum of the metric values for each path $p$ from the source $s$ to each leaf $s \in S$, is less than a chosen limit $\Delta$. ST and CST are NP-Complete problems [Winter 1987] since the second can be reduced to the first one.

As can be seen, the problems related to multicast inherit both the difficulty of multiple constrained metrics, and the difficulty to reach multiple end-nodes at the same time.

\subsection{Unicast Routing with QoS Extensions}

In Fig. 1 we show an example of a unicast communication between the source, generating data, and the only one receiver (i.e. the destination of the communication): the thick oriented lines highlight the direction of the packet flow, while dashed lines correspond to links not traversed.

Now we present some of the unicast QoS routing proposals, each of them oriented at optimizing only a small subset of the possible QoS metrics or using heuristics, since, as presented in Sec. 3.1, the problem is in general NP-Complete. For example, several solutions have been proposed for bandwidth-bounded routing: an interesting approach proposed in [Ma and Steenkiste 1997] exploits the dependencies among resources, e.g. available bandwidth, delay, and buffer space, to simplify the problem; then, a modified Bellman-Ford algorithm can be used to solve the problem. One approach to satisfy both bandwidth and delay bounds is to first prune all links not satisfying the bandwidth requirement. Dijkstras shortest path algorithm is then applied to find a feasible path, if any, satisfying the delay require- 
ments [Wang and Crowcroft 1996]. The problem of optimizing both the bandwidth and the delay can be either solved as a widest shortest path problem or a shortest widest path problem, depending if the algorithm gives higher priority to selecting paths with minimum hop counts (i.e. widest shortest path), or to selecting paths with maximum bandwidth (i.e. shortest widest path) [Wang and Crowcroft 1996]. The objective of multi-constrained routing is to simultaneously satisfy a set of constraints, as described in [Ma and Steenkiste 1997; Korkmaz and Krunz 2001]. In [Korkmaz and Krunz 2001] is proposed a heuristic approach for the multi-constrained optimal path problem (defined a $H_{-} M C O P$ ), which optimizes a non-linear function (for feasibility) and a primary function (for optimality). There are also solutions for bandwidth and cost bounded routing, which typically map the cost or the bandwidth to a bounded integer value, and then solve the problem in polynomial time using an extended version of Bellman-Ford or Dijkstra algorithms [Chen and Nahrstedt 1998].

\subsection{Multicast Routing with QoS extensions}

Multicast is an important bandwidth-conserving technology that reduces traffic by simultaneously delivering a single stream of information to multiple receivers (as shown in Fig. 2). Therefore, while saving resources, multicast is well suited to concurrently distribute contents on behalf of applications asking for a certain timeliness of delivery: thus, also multicast routing has naturally been extended to guarantee QoS requirements [Wang and Hou 2000]. In its simplest implementation, multicast can be provided using multiple unicast transmissions (i.e. the source would be in charge to open them), but with this solution, the same packet can traverse the same link multiple times, thus increasing the network traffic. For this reason, the network must provide this service natively, by creating multicast (group) addresses and by letting the routers duplicate the packet only when the distribution tree effectively forks. In this way, the source node has to know only one global address for all the destinations, and the network (i.e. the routers) can optimally "split" the flow towards the receivers, knowing also how to optimize traffic: the source node cannot have this information.

A multicast address is also called a multicast group address, with which the routers can locate and send packets to all the members in the group. A group member is a host that expresses interest in receiving packets sent to a specific group address. A group member is also sometimes called a receiver or a listener. A multicast source is a host that sends packets with the destination address set to a multicast group. To deliver data only to interested parties, routers in the network build a multicast (or distribution) tree (Fig. 2). Each subnetwork that contains at least one interested listener is a leaf of the tree. Where the tree branches, routers replicate the data and send a single packet down each branch. No link ever carries a duplicate flow of packets, since packets are replicated in the network only at the point where paths diverge, reducing the global traffic.

Multicast problem has been studied with several algorithms and variants, such as Shortest-Path Tree (SPT), MST, ST, CST (see Sec. 3), and other miscellaneous trees [Wang and Hou 2000]. Algorithms based on SPT (e.g. Dijkstra or BellmanFord [Cormen et al. 1990]) aim to minimize the sum of the weights on the links from the source to each receiver, and if all the link cost one unit, the resulting tree

ACM Journal Name, Vol. V, No. N, 20 YY. 


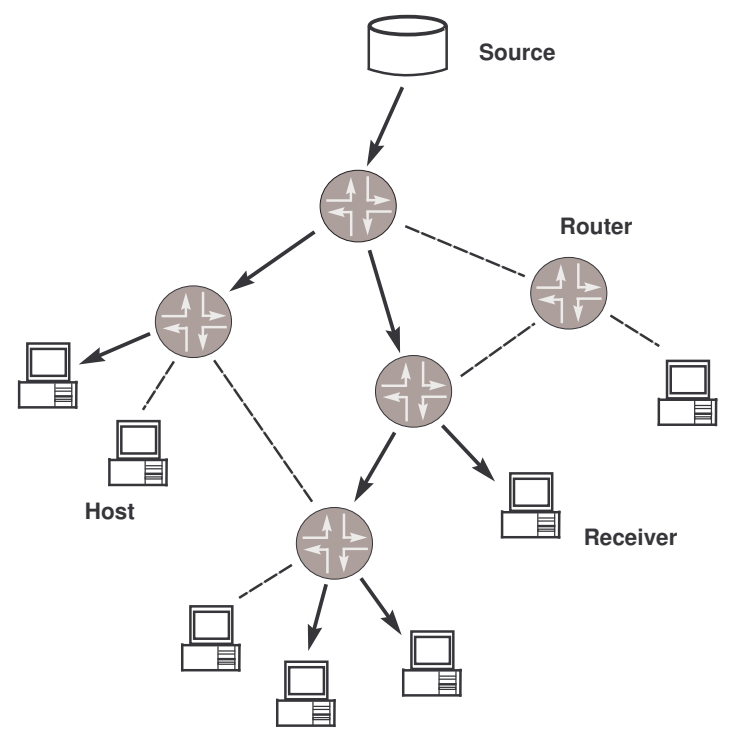

Fig. 2. An example of a multicast tree built over a network: oriented arcs highlight the tree (direction is down stream), while dashed lines correspond to links not traversed by the flow.

is the least-hop one.

Multicast QoS routing is generally more complex than unicast QoS routing, and for this reason less proposals have been elaborated in this area [Younis and Fahmy 2003; Paul and Raghavan 2002]. With respect to unicast, the additional complexity stems from the need to support shared and heterogeneous reservation styles (towards distinct group members) and global admission control of the distribution flow. Some of the approaches use a Steiner tree formulation [Berman et al. 1979] or extend existing algorithm to optimize the delay (i.e. MOSPF [Moy 1998] is the multicast version of the classical OSPF), while the Delay Variation Multicast Algorithm (DVMA) [Rouskas and Baldine 1997] computes a multicast tree with both bounded delay and bounded jitter. Also, delay-bounded and cost-optimized multicast routing can be formulated as a Steiner tree: an example approach is $Q o S$-aware Multicast Routing Protocol [Chen et al. 2000] (QMRP). Other multicast QoS routing algorithms and related problems (entailing stability, robustness and scalability) are presented in [Younis and Fahmy 2003].

\section{FINDING UNICAST QOS ROUTES WITH SCLP PROGRAMS}

In this Section we will show how to represent and solve unicast QoS routing with SCLP. At the beginning the problem will be treated only from the cost optimization view, i.e. as a SP problem, while in the last part we propose an example on how to add constraints on the path (i.e. solving the MCOP problem seen in Sec. 3.2). Sec. 4.1 translates SP problems as SCLP programs, while in Sec. 4.2 the same model is extended for multi-criteria optimizations, thus featuring vectors of costs on the edges, and not a single value. Sec. 4.3 describes the case where each arc also stores information about the modality to be used to traverse the arc. At last, 


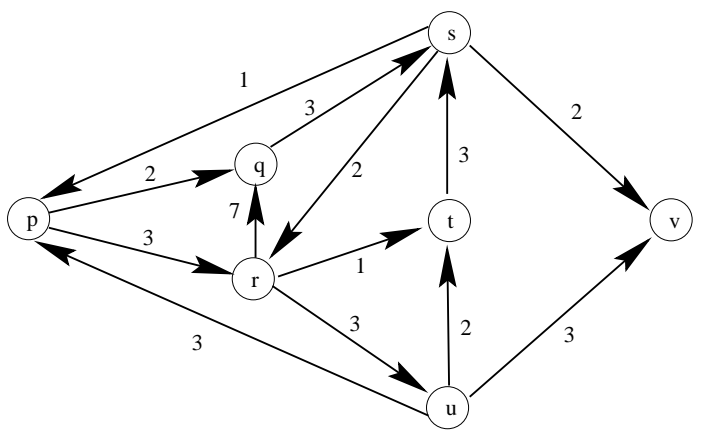

Fig. 3. An SP problem.

in Sec. 4.4 we add constraints on the QoS metrics, in order to fully obtain a model for constrained paths.

\subsection{From SP Problems to SCLP Programs}

We suppose to work with a graph $G=(N, E)$, where each oriented arc $e \in E$ from node $p$ to node $q(p, q \in N)$ has associated a label representing the cost of the arc from $p$ to $q$, as the example in Fig 3. This graph can be easily used to represent a network, if nodes are associated to network devices (routers and hosts) and arcs to network links. From any SP problem we can build an SCLP program as follows.

For each arc we have two clauses: one describes the arc and the other one its cost. More precisely, the head of the first clause represents the starting node, and its body contains both the final node and a predicate, say $c$, representing the cost of the arc. Then, the second clause is a fact associating to predicate $c$ its cost (which is a semiring element). Even if in this Section the concept of cost is quite general, we recall that with this fact we represent the QoS metric values on the arc (see Sec. 3). For example, if we consider the arc from $p$ to $q$ with cost 2 , we have the clause

$\mathrm{p}:-c_{p q}, \mathrm{q}$.

and the fact

$c_{p q}:-2$.

Finally, we must code that we want $v$ to be the final node of the path. This is done by adding a clause of the form $\mathrm{v}:-0$. Note also that any node can be required to be the final one, not just those nodes without outgoing arcs (like $v$ is in this example). The whole program corresponding to the SP problem in Fig. 3 can be seen in Table II.

To represent the classical version of SP problems, we consider SCLP programs over the semiring $S=\langle\mathbb{N}, \min ,+,+\infty, 0\rangle$, which is an appropriated framework to represent constraint problems where one wants to minimize the sum of the costs of the solutions. For example, we can imagine that the cost on the arcs represents to us the average delay experienced on the related link (measured in tens milliseconds). To compute a solution of the SP problem it is enough to perform a query in the SCLP framework; for example, if we want to compute the cost of the path from $r$ to $v$ we have to perform the query :- r. For this query, we obtain the value 
Table II. The SCLP program representing the SP problem in Fig. 3.
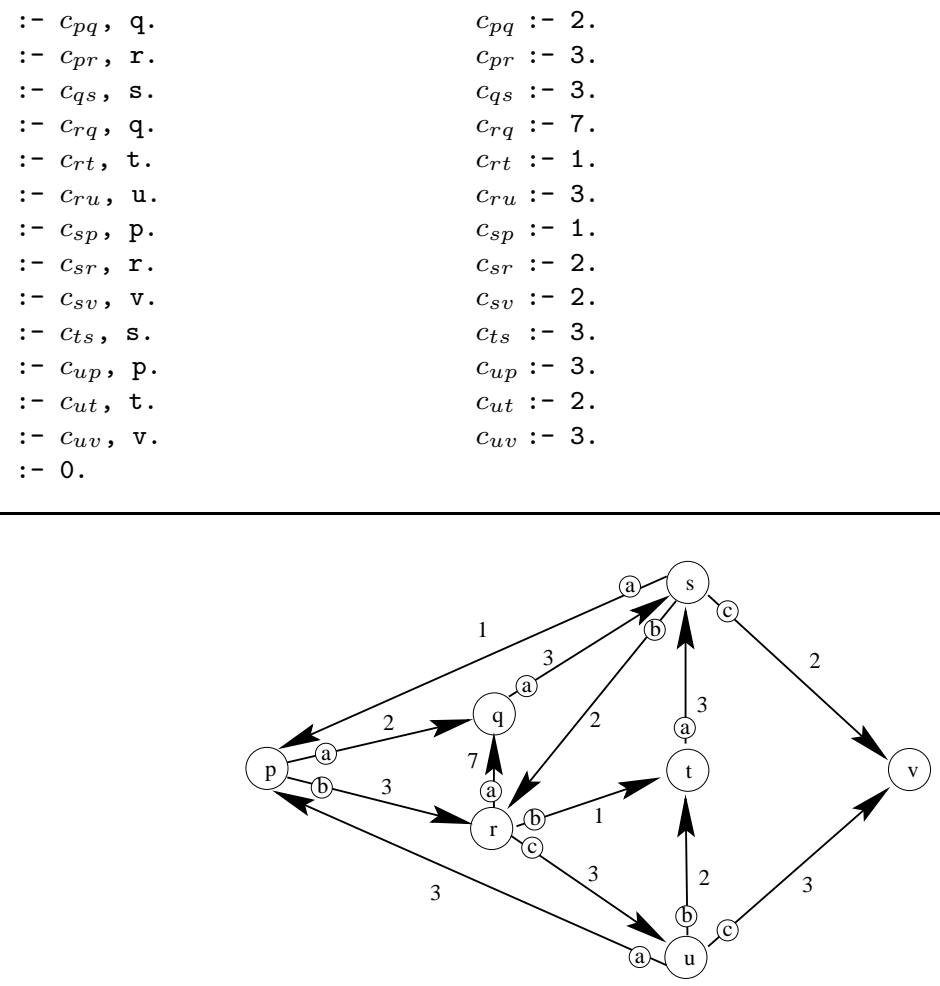

Fig. 4. An SP problem with labeled arcs.

6 , that represents the cost of the best path(s) from $r$ to $v$, optimizing in this way the total delay experienced on the route from $r$ to $v$. Clearly, different semirings can be chosen to represent the composition properties of the different metrics, as we will see better in Sec. 4.2 by proposing bandwidth as the second metric describing the link costs.

Notice that to represent classical SP problems in SCLP, we do not need any variable. Thus the resulting program is propositional. However, this program, while giving us the cost of the shortest paths, does not give us any information about the arcs which form such paths. This information could be obtained by providing each predicate with an argument, which represents the arc chosen at each step.

Figure 4 shows the same SP problem of Fig. 3 where the arcs outgoing each node have been labeled with different labels to distinguish them. Such labels can then be coded into the corresponding SCLP program to "remember" the arcs traversed during the path corresponding to a solution. For example, clause

$\mathrm{p}:-c_{p q}$, q.

would be rewritten as

$\mathrm{p}(\mathrm{a}):-c_{p q}, \mathrm{q}(\mathrm{X})$. 
Here constant $a$ represents one of the arcs going out of $p$ : the one which goes to $q$. If all clauses are rewritten similarly, then the answer to a goal like :- $r(X)$ will be both a semiring value (in our case 6 ) and a substitution for $X$. This substitution will identify the first arc of a shortest path from $r$ to $v$. For example, if we have $X=b$, it means that the first arc is the one that goes from $r$ to $t$. To find a complete shortest path, we just need to compare the semiring values associated with each instantiated goal, starting from $r$ and following the path. For example, in our case (of the goal $\exists X . r(X)$ ) we have that the answer to the goal will be $X=c$ with semiring value 6 . Thus we know that a shortest path from $r$ to $v$ can start with the arc from $r$ to $u$. To find the following arc of this path, we compare the semiring values of $u(a), u(b)$, and $u(c)$. The result is that $u(c)$ has the smallest value, which is 3 . Thus the second arc of the shortest path we are constructing is the one from $u$ to $v$. The path is now finished because we reached $v$ which is our final destination.

Notice that a shortest path could be found even if variables are not allowed in the program, but more work is needed. In fact, instead of comparing different instantiations of a predicate, we need to compare the values associated with the predicates that represent nodes reachable by alternative arcs starting from a certain node, and sum them to the cost of such arcs. For example, instead of comparing the values of $p(a)$ and $p(b)$ (Fig. 4), we have to compare the values of $q+2$ and of $r+3$ (Fig. 3).

A third alternative to compute a shortest path, and not only its cost, is to use lists: by replacing each clause of the form

$\mathrm{p}:-\mathrm{c}_{x y}, \mathrm{q}$.

with the clause

$\mathrm{p}([\mathrm{a} \mid \mathrm{T}]):-\mathrm{c}_{x y}, \mathrm{q}(\mathrm{T})$.

during the computation we also build the list containing all arcs which constitute the corresponding path. Thus, by giving the goal :- p(L)., we would get both the cost of a shortest path and also the shortest path itself, represented by the list $L$.

An alternative representation, probably more familiar for CLP users, of SP problems in SCLP is one where there are facts of the form

$c(p, q):-2$.

:

$\mathrm{c}(\mathrm{u}, \mathrm{v}):-3$.

to model the graph, and the two clauses

$\operatorname{path}(\mathrm{X}, \mathrm{Y}):-\mathrm{c}(\mathrm{X}, \mathrm{Y})$.

$\operatorname{path}(\mathrm{X}, \mathrm{Y}):-\mathrm{c}(\mathrm{X}, \mathrm{Z}), \operatorname{path}(\mathrm{Z}, \mathrm{Y})$.

to model paths of length one or more. In this representation the goal to be given to

find the cost of the shortest path from $p$ to $v$ is :- path $(\mathrm{p}, \mathrm{v})$. This representation is obviously more compact than the one in Table II, and has equivalent results and properties. However, in next Sections we will continue using the simpler representation, used in Table II, where all the clauses have at most one predicate in the body. The possibility of representing SP problems with SCLP programs containing only such a kind of clauses is important, since it will allow us to use efficient algorithms to compute the semantics of such programs (see [Bistarelli et al. 2002] for more details).

ACM Journal Name, Vol. V, No. N, 20 YY. 


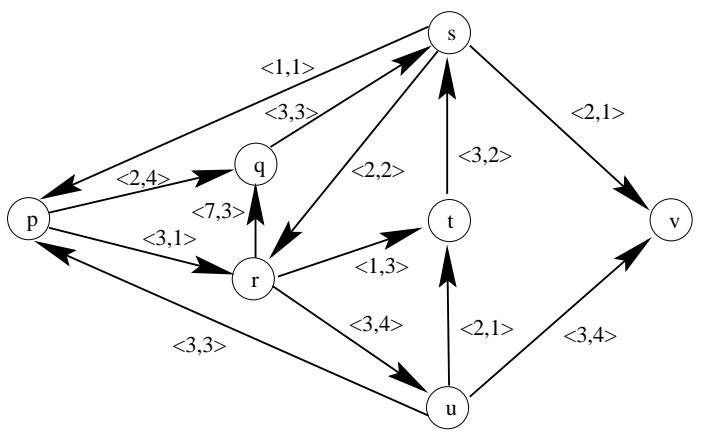

Fig. 5. A multi-criteria SP problem.

\subsection{Partially-Ordered SP Problems}

Sometimes, the costs of the arcs are not elements of a totally ordered set. A typical example is obtained when we consider multi-criteria SP problems. Consider for example the multi-criteria SP problem shown in Fig. 5: each arc has associated a pair that represent the weight of the arc in terms of cost of use and average delay (i.e. two possible QoS metrics); thus, the values are in the $\langle$ cost, delay $\rangle$ form. Given any node $p$, we want to find a path from $p$ to $v$ (if it exists) that minimizes both criteria. In this example, there may be cases in which the labels of two arcs are not compatible, like $\langle 5,20\rangle$ and $\langle 7,15\rangle$, since the cost is better in the first pair, while the delay is lower in the second one. In general, when we have a partially ordered set of costs, it may be possible to have several paths, all of which are not dominated by others, but which have different incomparable costs (see also Sec. 6).

We can translate this SP problem in Fig. 5 into the corresponding SCLP program in Table III. This program works over the semiring

$$
\left\langle\mathbb{N}^{2}, \min ',{ }^{\prime},\langle+\infty,+\infty\rangle,\langle 0,0\rangle\right\rangle,
$$

where $\min ^{\prime}$ and $+^{\prime}$ are classical $\min$ and + , suitably extended to pairs. In practice, this semiring is obtained by putting together, via the Cartesian product, two instances of the semiring $\langle\mathbb{N}, \min ,+,+\infty, 0\rangle$ (we recall that the Cartesian product of two c-semirings is a c-semiring as well [Bistarelli et al. 1997b]). One of the two instances is used to deal with the cost criteria, the other one is for the delay criteria. By working on the combined semiring, we can deal with both criteria simultaneously: the partial order will tell us when a $\langle$ cost, delay $\rangle$ pair is preferable to another one, and also when they are not comparable.

To give an idea of another practical application of partially-ordered SP problems, just think of network routing problems where we need to optimize according to the following criteria: minimize the delay, minimize the cost, minimize the number of arcs traversed, and maximize the bandwidth. The first three criteria correspond to the same semiring, which is $\langle\mathbb{N}, \min ,+,+\infty, 0\rangle$, while the fourth criteria can be characterized by the semiring $\langle\mathcal{B}, \max , \min , 0,+\infty\rangle$, where $\mathcal{B}$ is the set of the possible bandwidth values (in Sec. 5.1 we will better investigate these semirings). In this example, we have to work on a semiring which is obtained by vectorizing all these four semirings. Each of the semirings is totally ordered but the resulting 
Table III. The SCLP program representing the multi-criteria SP problem in Fig. 5.

\begin{tabular}{|c|c|}
\hline$:-c_{p q}, \mathrm{q}$ & $c_{p q}:-<2,4>$. \\
\hline$:-c_{p r}, \mathrm{r}$ & $c_{p r}:-<3,1>$. \\
\hline$:-c_{q s}, \mathrm{~s}$ & $c_{q s}:-<3,3>$. \\
\hline$:-c_{r q}, \mathrm{q}$ & $c_{r q}:-<7,3>$. \\
\hline$:-c_{r t}, \mathrm{t}$ & $c_{r t}:-\langle 1,3\rangle$. \\
\hline$:-c_{r u}$, u. & $c_{r u}:-<3,4>$. \\
\hline$:-c_{s p}, \mathrm{p}$ & $c_{s p}:-<1,1>$. \\
\hline$:-c_{s r}, \mathrm{r}$ & $c_{s r}:-<2,2>$. \\
\hline$:-c_{s v}, \mathrm{v}$ & $c_{s v}:-<2,1>$. \\
\hline$:-c_{t s}, \mathbf{s}$ & $c_{t s}:-<3,2>$. \\
\hline$:-c_{u p}, \mathrm{p}$. & $c_{u p}:-\langle 3,3\rangle$. \\
\hline$:-c_{u t}, \mathrm{t}$ & $c_{u t}:-<2,1>$. \\
\hline$:-c_{u v}, \mathrm{v}$ & $c_{u v}:-<3,4>$. \\
\hline
\end{tabular}

semiring, whose elements are four-tuples, is partially ordered.

\subsection{Modality-based SP Problems}

Until now we have considered situations in which an arc is labeled by its cost, be it one element or a tuple of elements as in the multi-criteria case. However, sometimes it may be useful to associate with each arc also information about the modality to be used to traverse the arc.

For example, interpreting the arcs of a graph as links between cities, we may want to model the fact that we can cover such an arc by car, or by train, or by plane. Another example of a modality could be the time of the day in which we cover the arc, like morning, afternoon, and night. One more example, this time strictly related to topic of this paper, could be represented by the modalities associated with the network link, e.g. wired, wireless or VPN, if there is the opportunity to establish a Virtual Private Network on it. Therefore the modalities can be used to manage policies for the routing (i.e. for policy routing). In all these examples, the cost of an arc may depend on its modality.

An important thing to notice is that a path could be made of arcs which not necessarily are all covered with the same modality. For example, the network connection between two distant buildings of the same company can be made of many hops, some of which are covered with the wireless modality and others with wired one. Moreover, it can be that different arcs have different sets of modalities. For example, from node $n_{0}$ to node $n_{1}$ we can use both the wired or wireless connection, and from node $n_{1}$ to node $n_{2}$ we can use only a VPN. Thus modalities cannot be simply treated by selecting a subset of arcs (all those with the same modality).

An example of an SP problem with three modalities representing a network with cryptographic service on the links $(c)$ (both wired or wireless), wired/no-crypt $(w)$, and wireless/no-crypt $(l)$ can be seen in Fig. 6. Here the problem is to find a shortest path from any node to $v$ (our final destination), and to know both its delay and also the modalities of its arcs. This SP problem can be modeled via the SCLP program in Table IV. In this program, the variables represent the modalities. 


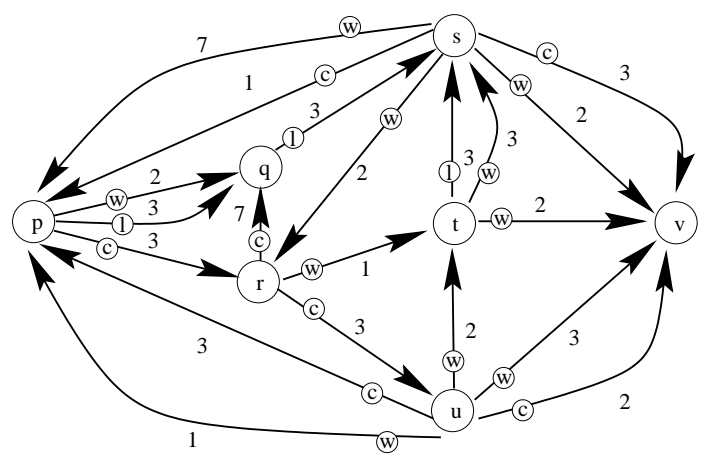

Fig. 6. An SP problem with modalities.

Table IV. The SCLP program representing the SP problem with modalities in Fig. 6.

$\begin{array}{ll}\mathrm{p}(\mathrm{X}) & :-c_{p q}(\mathrm{X}), \mathrm{q}(\mathrm{X}) . \\ \mathrm{p}(\mathrm{X}) & :-c_{p r}(\mathrm{X}), \mathrm{r}(\mathrm{X}) . \\ \mathrm{q}(\mathrm{X}) & :-c_{q s}(\mathrm{X}), \mathrm{s}(\mathrm{X}) . \\ \mathrm{r}(\mathrm{X}) & :-c_{r q}(\mathrm{X}), \mathrm{q}(\mathrm{X}) . \\ \mathrm{r}(\mathrm{X}) & :-c_{r t}(\mathrm{X}), \mathrm{t}(\mathrm{X}) . \\ \mathrm{r}(\mathrm{X}) & :-c_{r u}(\mathrm{X}), \mathrm{u}(\mathrm{X}) . \\ \mathrm{s}(\mathrm{X}) & :-c_{s p}(\mathrm{X}), \mathrm{p}(\mathrm{X}) . \\ \mathrm{s}(\mathrm{X}) & :-c_{s r}(\mathrm{X}), \mathrm{r}(\mathrm{X}) . \\ \mathrm{s}(\mathrm{X}) & :-c_{s v}(\mathrm{X}), \mathrm{v}(\mathrm{X}) . \\ \mathrm{t}(\mathrm{X}) & :-c_{t s}(\mathrm{X}), \mathrm{s}(\mathrm{X}) . \\ \mathrm{u}(\mathrm{X}) & :-c_{u p}(\mathrm{X}), \mathrm{p}(\mathrm{X}) . \\ \mathrm{u}(\mathrm{X}) & :-c_{u t}(\mathrm{X}), \mathrm{t}(\mathrm{X}) . \\ \mathrm{u}(\mathrm{X}) & :-c_{u v}(\mathrm{X}), \mathrm{v}(\mathrm{X}) . \\ \mathrm{v}(\mathrm{X}) & :-0 .\end{array}$

$$
\begin{aligned}
& c_{p q}(\mathrm{w}):-2 \text {. } \\
& c_{p q}(1):-3 \text {. } \\
& c_{p r} \text { (c) : }-3 \text {. } \\
& c_{q s}(1):-3 \text {. } \\
& c_{r q}(\mathrm{c}):-7 \text {. } \\
& c_{r t}(\text { w) }:-1 . \\
& c_{r u}(\mathrm{c}):-3 \text {. } \\
& c_{s p}(\mathrm{c}):-1 \text {. } \\
& c_{s p}(\mathrm{w}):-7 . \\
& c_{s r}(\mathrm{w}):-2 \text {. } \\
& c_{s v}(\mathrm{w}):-2 \text {. } \\
& c_{s v}(\mathrm{c}):-3 \text {. } \\
& c_{t s}(1):-3 \text {. } \\
& c_{t s}(\mathrm{w}):-3 . \\
& c_{u p}(\mathrm{c}):-3 \text {. } \\
& c_{u p} \text { (w) :- } 1 \text {. } \\
& c_{u t}(\mathrm{w}):-2 \text {. } \\
& c_{u v}(\mathrm{w}):-3 . \\
& c_{u v}(\mathrm{c}):-2 \text {. }
\end{aligned}
$$

If we ask the query :-p(c)., it means that we want to know the smallest delay for a route from $p$ to $v$ using the links with the cryptographic service. The result of this query in our example is $p(c)=8$ (using the path $p-r-u-v$ ).

Notice that the formulation shown in Fig. IV puts some possibly undesired constraints on the shortest path to be found. In fact, by using the same variable in all the predicates of a rule, we make sure that the same modality (in our case the same transport mean) is used throughout the whole path. If instead we want to allow different modalities in different arcs of the path, then we just need to change the rules by putting a new variable on the last predicate of each rule. For example, the rule in Tab. IV

$\mathrm{p}(\mathrm{X}):-c_{p q}(\mathrm{X}), \mathrm{q}(\mathrm{X})$.

would become

$\mathrm{p}(\mathrm{X}):-c_{p q}(\mathrm{X}), \mathrm{q}(\mathrm{Y})$. 
Now we can use a modality for the arc from $p$ to $q$, and another one for the next arc. In this new program, asking the query : $-p(c)$. means that we want to know the smallest delay for a trip from $p$ to $v$ using the cryptographic service in the first arc.

The same methods used in the previous Sections to find a shortest path, or a non-dominated path in the case of a partial order, can be used in this kind of SCLP programs as well. Thus we can put additional variables in the predicates to represents alternative arcs outgoing the corresponding nodes, and we can shift to the semiring containing sets of costs to find a non-dominated path. In particular, a clause like

$\mathrm{p}(\mathrm{X}):-c_{p q}(\mathrm{X}), \mathrm{q}(\mathrm{Y})$.

would be rewritten as

$\mathrm{p}(\mathrm{X}, \mathrm{a}):-c_{p q}(\mathrm{X}), \mathrm{q}(\mathrm{Y}, \mathrm{Z})$.

\subsection{Adding constraints to SP problems}

As seen in Sec. 3.1 a MCOP is much more difficult to solve than a SP problem, that is NP-Complete. So far we considered only variants of SP problems (partiallyordered or modality-based), but our aim is to provide a complete model for the unicast QoS routing. Thus, besides achieving cost optimization, we need also to consider constraints on the QoS metrics.

In our example we consider again the multi-criteria graph in Fig. 5: each arc has associated a pair that can represent the weight of the arc in terms of cost of use and average delay. However, in this case our goal is to minimize the cost and to guarantee a final average delay less than or equal to $8(80 \mathrm{msec})$, thus we want to add the boolean constraint delay $\leq 8$.

We chose to represent constrained paths with a program in CIAO Prolog [Bueno et al. 1997], a system that offers a complete Prolog system supporting ISO-Prolog, but, at the same time its modular design allows both restricting and extending the basic language. CIAO Prolog has also a fuzzy extension, but since it does not completely conform to the semantic of SCLP defined in [Bistarelli et al. 1997a] (due to interpolation in the interval of the fuzzy set), we decided to use the CIAO operators among constraints (as $<$ and $\leq$ ), and to model the $\times$ operator of the c-semiring with them. For this reason, we inserted the cost of the edges in the head of the clauses, differently from SCLP clauses which have the cost in the body of the clause. Similar reification processes have been already accomplished also in other works [Régin et al. 2000].

In Tab. $\mathrm{V}$ is shown the CIAO program that represents the graph in Fig. 5: here the edges (i.e. all the Edges facts in Table V) are in the form:

$$
\text { edge(Source_Node,Destination_Node, }[\text { Link_Cost,Link_Delay }])
$$

Moreover, we can see the two clauses that describe the structure of paths: Rule 1 and Rule 2 respectively represent the base (or termination) case, where a path is simply an edge, and the recursive case, needed to add one edge to the path. To avoid infinite recursion, and thus the program crashing, we need to deal with graph loops by considering the list of the already visited nodes, in order to prevent the search from visiting them twice. Moreover, we inserted a variable in the head of the

ACM Journal Name, Vol. V, No. N, 20 YY. 
Table V. The CIAO program representing all the paths of Fig. 5, with delay $\leq 8$

\begin{tabular}{|c|c|c|}
\hline$\stackrel{\mathscr{E}}{\underline{E}}$ & $\begin{array}{l}\text { times }([\mathrm{C} 1, \mathrm{D} 1],[\mathrm{C} 2, \mathrm{D} 2],[\mathrm{C} 3, \mathrm{D} 3 \\
\mathrm{C} 3=\mathrm{C} 1+\mathrm{C} 2 \\
\mathrm{D} 3=\mathrm{D} 1+\mathrm{D} 2\end{array}$ & \\
\hline $\begin{array}{l}\text { ச } \\
\text { 용 } \\
\text { யu }\end{array}$ & 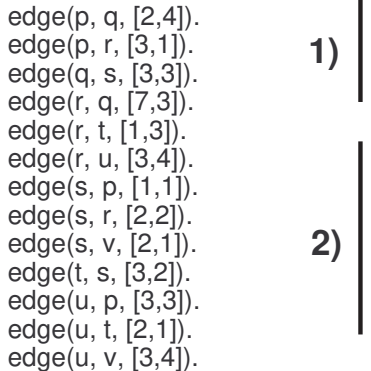 & $\begin{array}{l}\text { path }(X, Y,[X, Y],,[C, D], L):- \\
\text { edge }(X, Y,[C, D]), \\
D=<L . \\
\text { path }(X, Y,[X \mid T], V,[C, D], L):- \\
\text { edge }(X, Z,[C 1, D 1]), \\
\text { nocontainsX(V,Z), } \\
\text { path(Z,Y,T,[Z|V],[C2,D2],L), } \\
\text { times([C1,D1], [C2,D2], [C,D]), } \\
D=<L .\end{array}$ \\
\hline
\end{tabular}

path clauses to remember, at the end, all the visited nodes of the path: this list will store the nodes following the correct ordering of the visit. Finally, the last variable of the clause head is used to retrieve only the paths with a total delay equal or less than the passed value. Thus, the path clause-heads are in the form:

$$
\begin{gathered}
\operatorname{path}(\text { Source_Node,Destination_Node, Path_Nodes, Already_Visisted_Nodes, } \\
\left.[\text { Path_Cost,Path_Delay }], P a t h \_M a x \_D e l a y\right)
\end{gathered}
$$

The Aggregator clause mimics the $\times$ operation of the semiring (i.e. + extended to pairs, as in Sec. 4.2), and therefore it composes the global costs of the edges together, edge costs with costs, and edge delays with delays.

All the paths with a delay $\leq 8$, and the relative query $\operatorname{path}(p, v, P,[p],[C, D], 8)$ are shown in Fig. 10. The $p$ source node of the path, must be included in the list of the visited nodes from the beginning. Figure 10 corresponds to the output of the CIAO program in Tab. V, and for each of the three found paths it shows the variable $P$, which stores the sequence of the nodes in the path, and the $C-D$ pair, which corresponds to the total cost of the path in terms of $\langle$ cost, delay $\rangle$.

We remark the expressivity of the framework, since boolean constraints can be easily added to the query instead of being directly hard coded in the program. For example, with a query like $\operatorname{path}(p, v, P,[p],[C, D]), D<8$ returns all the paths with a delay value less than 8 .

\section{EXTENDING THE MODEL TO DEAL WITH MULTICAST QOS ROUTING}

Now we extend the framework given in Sec. 4 in order to manage also the multicast delivery schema. The first step is represented by the use of hypergraphs instead of simple graphs, since we need a method to connect one node to multiple destinations at the same time (i.e. when the same packet must be routed on different links). 


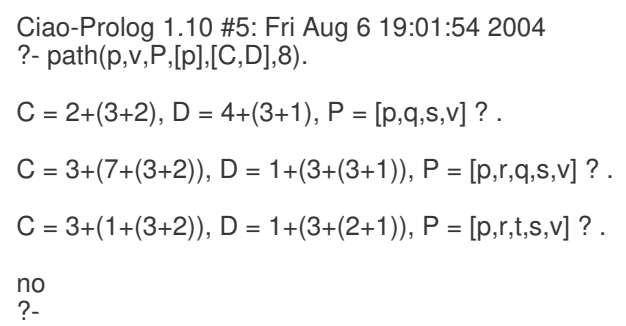

Fig. 7. The CIAO output for the program in Tab. V: three paths are found with delay $\leq 8$.

Section 5.1 presents a possible transformation procedure from networks to and-or graphs, showing also how to find a cost for the hyperarcs and related semirings. In Sec. 5.2 we describe the SCLP programs representing and solving the multicast QoS routing. In Sec. 5.3 we associate modalities to hyperarcs, as we did in Sec. 4.3 for paths.

\subsection{From networks to hypergraphs}

In this Section we explain a method to translate the representation of a multicast network with QoS requirements (Fig. 9a) into a corresponding weighted and-or graph [Martelli and Montanari 1978] (Fig. 9b). This procedure can be split in three distinct steps, respectively focusing on the representation of $i$ ) network nodes, ii) network links and iii) link costs in terms of QoS metrics.

An and-or graph [Martelli and Montanari 1978] is defined essentially as a hypergraph. Namely, instead of arcs connecting pairs of nodes there are hyperarcs connecting an $n$-tuple of nodes $(n=1,2,3, \ldots)$. Hyperarcs are called connectors and they must be considered as directed from their first node to all others. Formally an and-or graph is a pair $G=(N, C)$, where $N$ is a set of nodes and $C$ is a set of connectors

$$
C \subseteq N \times \bigcup_{i=0}^{k} N^{i} .
$$

Note that the definition allows 0-connectors, i.e. connectors with one input and no output node. 0-connectors are represented as a line ending with a square (Fig. $9 b$ ). In the following of the explanation we will also use the concept of and tree [Martelli and Montanari 1978]: given an and-or graph $G$, an and tree $H$ is a solution tree of $G$ with start node $n_{r}$, if there is a function $g$ mapping nodes of $H$ into nodes of $G$ such that:

- the root of $H$ is mapped in $n_{r}$.

-if $\left(n_{i_{0}}, n_{i_{1}}, \ldots, n_{i_{k}}\right)$ is a connector of $H$, then $\left(g\left(n_{i_{0}}\right), g\left(n_{i_{1}}\right), \ldots, g\left(n_{i_{k}}\right)\right)$ is a connector of $G$.

In words, a solution tree of an and-or graph is analogous to a path of an ordinary graph: it can be obtained by selecting exactly one outgoing connector for each node.

Each of the network nodes can be easily cast in the corresponding and-or graphs as a single graph node: thus, each node in the graph can represent an intercon- 


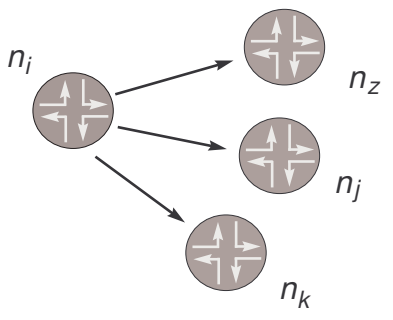

a)

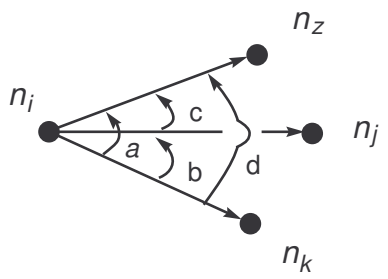

b)

Fig. 8. a) the f-star of $n_{i}$ network-node and $b$ ) its representation with connectors.

necting device (e.g. a router), or a node acting as the source of a multicast communication (injecting packets in the network), or, finally, a receiver belonging to a multicast group and participating in the communication. In Sec. 5.2, when we will look for the best tree solution, the root of the best and tree will be mapped to the node representing the source of the multicast communication; in the same way, receivers will be modelled by the leaves of the resulting and tree. When we translate a receiver, we add an outgoing 0-connector to model the end-point of the communication, and whose cost will be explained below. Suppose that $\left\{n_{0}, n_{1}, \ldots, n_{9}\right\}$ in Fig. $9 a$ are the identifiers of the network nodes.

To model the links, we examine the forward star $(f$-star $)$ of each node in the network (i.e. the set of arcs outgoing from a node): we consider the links as oriented, since the cost of sending packets from node $n_{i}$ to $n_{j}$ can be different from the cost of sending from $n_{j}$ to $n_{i}$ (one non-oriented link can be easily replaced by two oriented ones). Supposing that the f-star of node $n_{i}$ includes the $\operatorname{arcs}\left(n_{i}, n_{j}\right)$, $\left(n_{i}, n_{k}\right)$ and $\left(n_{i}, n_{z}\right)$, we translate this f-star by constructing one connector directed from $n_{i}$ to each of the subsets of destination nodes $\{j, k, z\}$ (Fig. 8), for a possible maximal number of $2^{|N|}-1$ subsets (where $|N|$ is the cardinality of the set of node in the graph), i.e. excluding the emptyset; in Sec. 8 we will see how to minimize this exponential growth. Thus, all the resulting connectors with $n_{i}$ as the input node are $\left(n_{i}, n_{j}\right),\left(n_{i}, n_{k}\right),\left(n_{i}, n_{z}\right),\left(n_{i}, n_{k}, n_{j}\right),\left(n_{i}, n_{k}, n_{z}\right),\left(n_{i}, n_{j}, n_{z}\right)$ and $\left(n_{i}, n_{j}, n_{k}, n_{z}\right)$. In the connectors tuple-ordering of the nodes, the input node is at the first position and the output nodes (when more than one) follow the orientation of the related arrow in Fig. 8.

To simplify Fig. $8 b$, the arcs linking directly two nodes represent 1-connectors $\left(n_{i}, n_{j}\right),\left(n_{i}, n_{k}\right)$ and $\left(n_{i}, n_{z}\right)$, while curved oriented lines represent $n$-connectors (with $n>1$ ), where the set of their output nodes corresponds to the output nodes of the traversed arcs. With respect to $n_{i}$, in Fig. 8 we have a curved line labelled with $a$ that corresponds to $\left(n_{i}, n_{k}, n_{j}, n_{z}\right), b$ to $\left(n_{i}, n_{k}, n_{j}\right), c$ to $\left(n_{i}, n_{j}, n_{z}\right)$, and, at last, $d$ to $\left(n_{i}, n_{k}, n_{z}\right)$. To have a clear figure, the network links in Fig. $9 a$ are oriented "towards" the receivers, thus we put only the corresponding connectors in Fig. $9 b$.

In the example we propose here, we are interested in QoS link-state information concerning only bandwidth and cost. Therefore, each link of the network can be labeled with a 2-dimensional cost, for example the pair $\langle 7,3\rangle$ tells us that the 
maximum bandwidth on that specific link is $70 \mathrm{Mbps}$ and the cost is $30 €$. In general, we could have a cost expressed with a $n$-dimensional vector, where $n$ is the number of metrics to be taken in account while computing the best distribution tree. Since we want to maintain this link state information even in the and-or graph, we label the corresponding connector with the same tuple of values (Fig. 9).

In the case when a connector represent more than one network link (i.e. a $n$ connector with $n \geq 2$ ), its cost is decided by assembling the costs of the these links with the composition operation $\circ$, which takes as many $n$-dimensional vectors as operands, as the number of links represented by the connector. Naturally, we can instantiate this operation for the particular types of costs adopted to express QoS: for the example given in this Section, the result of $\circ$ is the minimum bandwidth and the highest cost (it could be also the sum of all the costs of the links), ergo, the worst QoS metric values:

$$
\circ\left(\left\langle b_{1}, c_{1}\right\rangle,\left\langle b_{2}, c_{2}\right\rangle, \ldots,\left\langle b_{n}, c_{n}\right\rangle\right) \longrightarrow\left\langle\min \left(b_{1}, b_{2}, \ldots, b_{n}\right), \max \left(c_{1}, c_{2}, \ldots, c_{n}\right)\right\rangle
$$

The cost of the connector $\left(n_{1}, n_{3}, n_{4}\right)$ in Fig. $9 b$ will be $\langle 7,3\rangle$, since the costs of connectors $\left(n_{1}, n_{3}\right)$ and $\left(n_{1}, n_{4}\right)$ are respectively $\langle 7,2\rangle$ and $\langle 10,3\rangle$ :

$$
\circ(\langle 7,2\rangle,\langle 10,3\rangle)=\langle 7,3\rangle
$$

To simplify Fig. 9b, we inserted only the costs for the 1-connectors, but the costs for the other connectors can be easily computed with the o operation, and are all reported in Tab. VI.

So far, we are able to translate an entire network with QoS requirements in a corresponding and-or weighted graph, but still we need some algebraic framework to model our preferences for the links to use in the best tree. For this reason, we use the semiring structure (Sec. 2). An exhaustive explanation of the semiring framework approach for shortest-distance problems is presented in [Mohri 2002; Tarjan 1979].

For example, if we are interested in maximizing the bandwidth of the distribution tree, we can use the semiring $S_{\text {Bandwidth }}=\langle\mathcal{B} \cup\{0,+\infty\}$, max, $\min , 0,+\infty\rangle$; otherwise, we could be interested in minimizing the global bandwidth with $\langle\mathcal{B} \cup$ $\{0,+\infty\}$, max, min, $+\infty, 0\rangle$, if our intention is to use an already busy link in order to preserve other unloaded links for future use (i.e. for traffic engineering purposes). We can use $S_{\text {Money }}=\langle\mathbb{N}$, min $,+,+\infty, 0\rangle$ for the money cost, if we need to minimize the total cost of the tree. Elements of $\mathcal{B}$ (i.e. the set of bandwidth values) can be obtained by collecting information about the network configuration, the current traffic state and technical information about the links. Since the composition of c-semirings is still a c-semiring [Bistarelli et al. 1997b],

$$
S_{N e t w o r k}=\left\langle\langle\mathcal{B} \cup\{0,+\infty\}, \mathbb{N}\rangle,+^{\prime}, \times^{\prime},\langle 0,+\infty\rangle,\langle+\infty, 0\rangle\right\rangle
$$

where $+^{\prime}$ and $\times^{\prime}$ correspond to the vectorization of the + and $\times$ operations in the two c-semirings: given $b_{1}, b_{2} \in \mathcal{B} \cup\{0,+\infty\}$ and $c_{1}, c_{2} \in \mathbb{N}$,

$$
\begin{gathered}
\left\langle b_{1}, c_{1}\right\rangle+^{\prime}\left\langle b_{2}, c_{2}\right\rangle=\left\langle\max \left(b_{1}, b_{2}\right), \min \left(c_{1}, c_{2}\right)\right\rangle \\
\left\langle b_{1}, c_{1}\right\rangle \times^{\prime}\left\langle b_{2}, c_{2}\right\rangle=\left\langle\min \left(b_{1}, b_{2}\right), c_{1}+c_{2}\right\rangle
\end{gathered}
$$

Clearly, the problem of finding best distribution tree is multi-criteria, since both bandwidth and cost must be optimized. We consider the criteria as independent

ACM Journal Name, Vol. V, No. N, 20YY. 


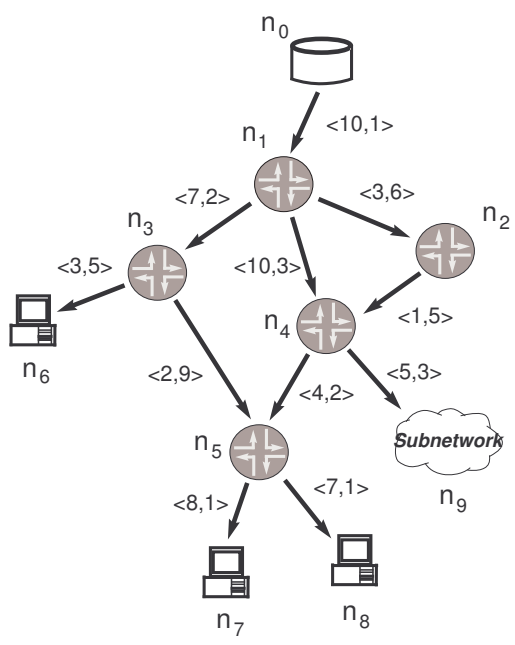

a)

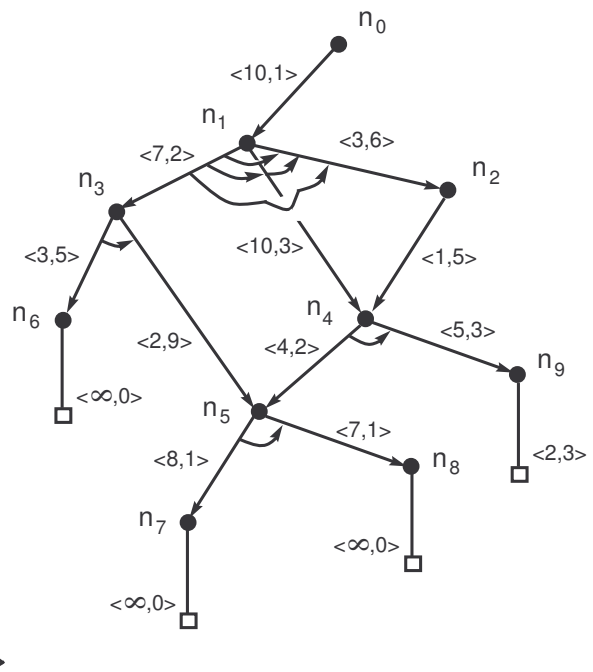

b)

Fig. 9. A network example and the corresponding and-or graph representation.

among them, otherwise they can be rephrased to a single criteria. Thus, the multidimensional costs of the connectors are not elements of a totally ordered set, and it may be possible to obtain several trees, all of which are not dominated by others, but which have different incomparable costs.

For each receiver node, the cost of its outgoing 0-connector will be always included in every tree reaching it. As a remind, a 0-connector has only one input node but no destination nodes. If we consider a receiver as a plain node, we can set the cost as the 1 element of the adopted c-semiring ( 1 is the unit element for $\times$ ), since the cost to reach this node is already completely described by the other connectors of the tree branch ending in this node: practically, we associate the highest possible QoS values to this 0 -connector, in this case infinite bandwidth and null cost. Otherwise we can imagine a receiver as a more complex subnetwork (as the node $n_{9}$ in Fig. 9), and thus we can set the cost of the 0-connector as the cost needed to finally reach a node in that subnetwork (as the cost $\langle 2,3\rangle$ for the 0 -connector after node $n_{9}$ in Fig. $9 b$ ), in case we do not want, or cannot, show the topology of the subnetwork, e.g. for security reasons.

\subsection{And-or graphs using SCLP}

In this Section, we represent an and-or graph with a program in SCLP. Using this framework, we can easily solve the multi-criteria example concerning the multicast QoS network in Fig. $9 b$.

As already proposed in Sec. 4, to represent the connectors in SCLP we can write clauses like $c\left(n_{i},\left[n_{j}, n_{k}\right]\right):-\langle 10,3\rangle$, stating that the graph has connector from $n_{i}$ to nodes $n_{j}$ and $n_{k}$ with a bandwidth cost of $100 \mathrm{Mbps}$ and a cost of $30 €$. Other SCLP clauses can properly describe the structure of the tree we desire to search 
over the graph.

For the same reasons exposed in Sec. 4.4, we choose to represent an and-or graph with a program in CIAO Prolog [Bueno et al. 1997]. As an example, from the weighted and-or graph problem in Fig. $9 b$ we can build the corresponding CIAO program of Table VI as follows. The set of network edges (or 1-connectors) is highlighted as Edges in Tab. VI. Each fact has the structure

$$
\text { edge(source_node, [dest_nodes], [bandwidth, cost]) }
$$

e.g. the fact edge $\left(n_{0},\left[n_{1}\right],[10,1]\right)$ represents the 1-connector of the graph $\left(n_{0}, n_{1}\right)$ with bandwidth equal to $100 \mathrm{Mbps}$ and cost $10 €$. The Rules 1 in Tab. VI are used to compose the edges (i.e. the 1-connectors) together in order to find all the possible $n$-connectors with $n \geq 1$, by aggregating the costs of 1 -connectors with the o composition operator, as described in Sec. 5.1 (the lowest of the bandwidths and the greatest of the costs of the composed 1-connectors). Therefore, with these clauses (in Rules 1) we can automatically generate the set of all the connectors outgoing from the considered node (in Table VI, nocontainsx and insert_last are CIAO predicates used to build a well-formed connector). The Leaves in Table VI represent the 0 -connectors (a value of 1000 represents $\infty$ for bandwidth). The plus and times rules in Table VI respectively mimic the + and $\times$ operations of the semiring proposed in Sec. 5.1: $S_{\text {Network }}=\left\langle\langle\mathcal{B} \cup\{0,+\infty\}, \mathbb{N}\rangle,+^{\prime}, \times^{\prime},\langle 0,+\infty\rangle,\langle+\infty, 0\rangle\right\rangle$, where $+^{\prime}$ is equal to $\langle\max , \min \rangle$ and $x^{\prime}$ is equal to $\langle\min ,+\rangle$, as defined in Sec. 5.1. At last, the rules 2-3-4-5 of Tab. VI describe the structure of the routes we want to find over the graph. Rule 2 represents a route made of only one leaf node, Rule 3 outlines a route made of a connector plus a list of sub-routes with root nodes in the list of the destination nodes of the connector, Rule 4 is the termination for Rule 5, and Rule 4 is needed to manage the junction of the disjoint sub-routes with roots in the list $[X \mid X s]$; clearly, when the list $[X \mid X s]$ of destination nodes contains more than one node, it means we are looking for a multicast route. When we compose connectors or trees (Rule 2 and Rule 5), we use the times rule to compose their costs together. In Rule 5, append is a CIAO predicate used to join together the lists of destination nodes, when the query asks for a multicast route. At last, the route predicate in Sec. 5.1 collects all the results for the query and finally returns the solution chosen with the help of the plus predicate.

Notice that the o operator describes in Sec. 5.1 is modeled with Prolog clauses inside Rule 5, when composing multiple 1-connectors connectors.

Notice also that the complexity of append predicates in Tab. VI can be reduced by using difference lists instead. However, see Sec. 8 for complexity considerations.

To make the program in Tab. VI as readable as possible, we omitted two predicates: the sort predicate, needed to order the elements inside the list of destinationnodes of connectors and trees (otherwise, the query route $\left(n_{0},\left[n_{6}, n_{7}, n_{8}, n_{9}\right],[B, C]\right)$ and route $\left(n_{0},\left[n_{9}, n_{7}, n_{8}, n_{6}\right],[B, C]\right)$ would produce different results), and the intersection predicate to check that multiple occurrences of the same node do not appear in the same list of destination nodes, if reachable with different connectors (otherwise, for example, the tree $n_{0},\left[n_{7}, n_{7}, n 8, n 9\right]$ would be a valid result).

To solve the and-or graph problem it is enough to perform a query in Prolog language: for example, if we want to compute the cost of all the trees rooted at $n_{0}$ and having as leaves the nodes representing all the receivers (i.e. $\left\{n_{6}, n_{7}, n_{8}, n_{9}\right\}$ ), 
Table VI. The CIAO program representing the best result tree over the weighted and-or graph problem in Fig. $9 b$.

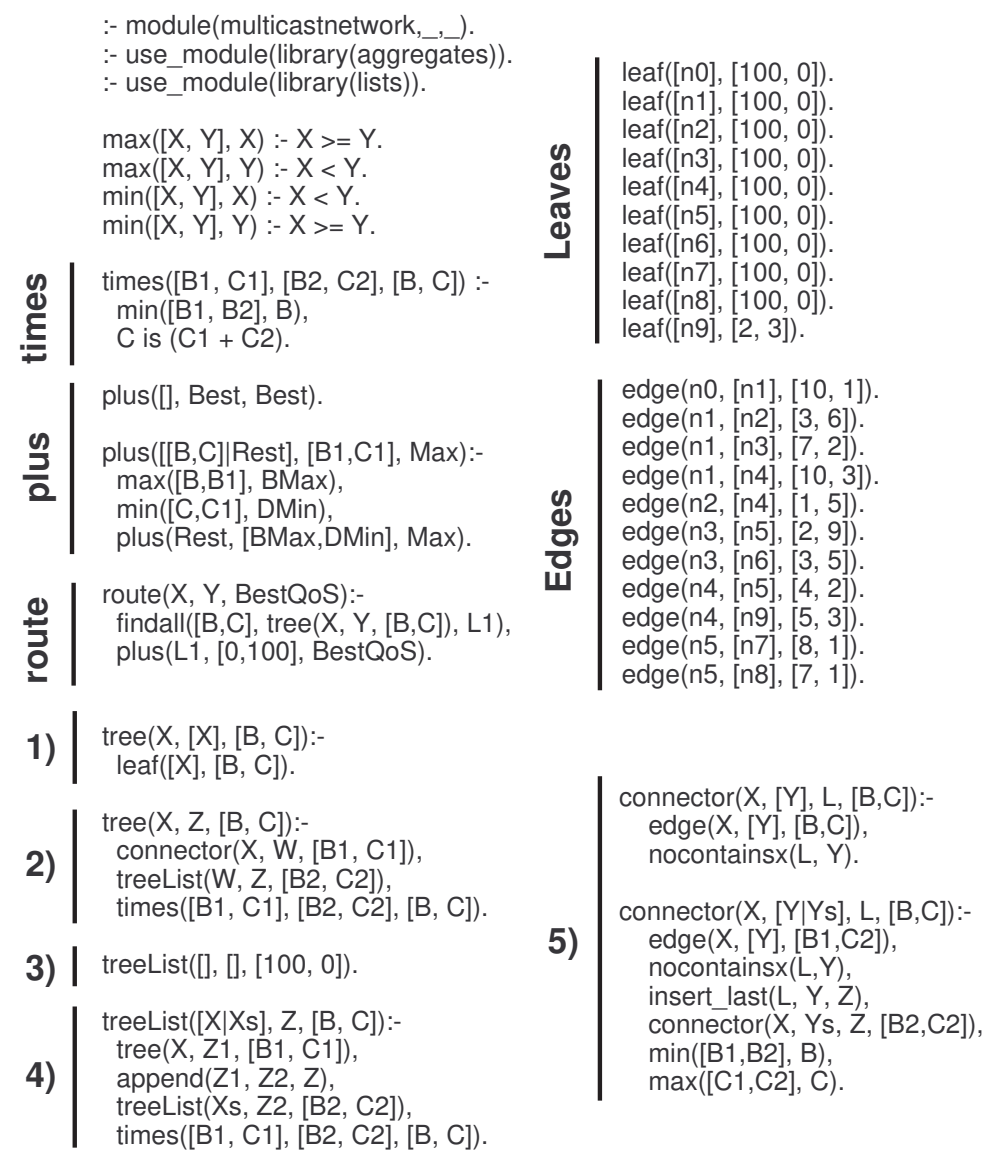

we have to perform the query route $\left(n_{0},\left[n_{6}, n_{7}, n_{8}, n_{9}\right],[B, C]\right)$, where $B$ and $C$ variables will be instantiated with the bandwidth and cost of the found trees. The output of the CIAO program for this query corresponds to the cost of the tree in Fig. 11, i.e. $\langle 2,16\rangle$. For this query, the output of the program in Tab. VI is shown in Fig. 10. The tree in Fig. 11 is a solution tree (see Sec. 5.1) for the graph in Fig. 9b, with mapping function $g: g\left(n_{0}^{\prime}\right)=n_{0}, g\left(n_{1}^{\prime}\right)=n_{1}, g\left(n_{3}^{\prime}\right)=n_{3}, g\left(n_{4}^{\prime}\right)=$ $n_{4}, g\left(n_{5}^{\prime}\right)=n_{5}, g\left(n_{6}^{\prime}\right)=n_{6}, g\left(n_{7}^{\prime}\right)=n_{7}, g\left(n_{8}^{\prime}\right)=n_{8}, g\left(n_{9}^{\prime}\right)=n_{9}$.

A global cost can be given to and trees: recursively, to every subtree of $H$ with root node $n_{i_{0}}$, a cost $c_{i_{0}}$ is given as follows:

-If $n_{i_{0}}$ is a leaf, then its cost is the associated constant.

- If $n_{i_{0}}$ is the input node of a connector $\left(n_{i_{0}}, n_{i_{1}}, \ldots, n_{i_{k}}\right)$, then its cost is $c_{i_{0}}=$ $f_{r}\left(c_{i_{1}}, \ldots, c_{i_{k}}\right)$ where $f_{r}$ is the function cost associated with the connector, and $c_{i_{1}}, \ldots, c_{i_{k}}$ are the costs of the subtrees rooted at nodes $n_{i_{1}}, \ldots, n_{i_{k}}$. 


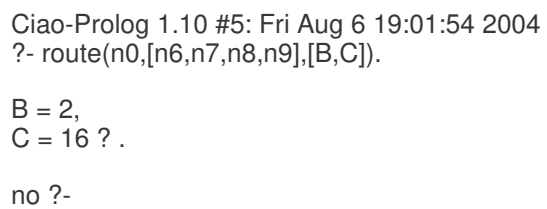

Fig. 10. The CIAO output for the program in Tab. V. The best bandwidth and delay values are found for the tree with $n_{6}, n_{7}, n_{8}, n_{9}$ destinations

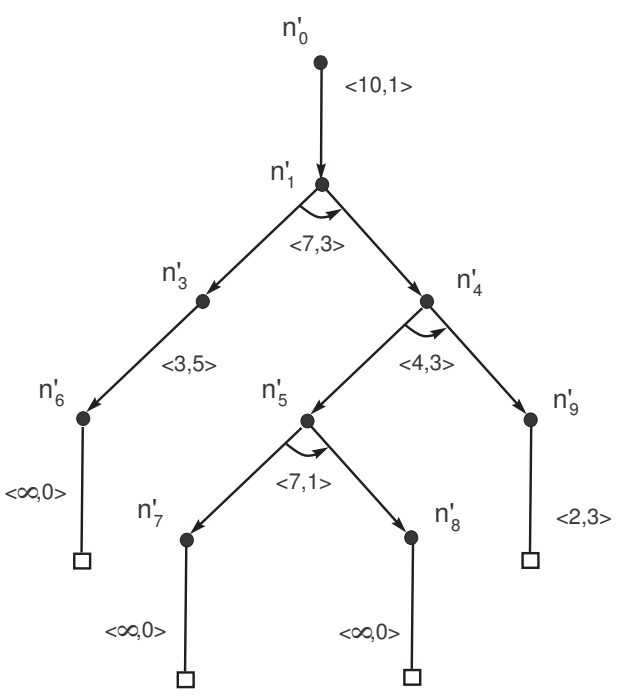

Fig. 11. The best multicast distribution tree that can be found with the program in Table VI.

The final cost of the tree in Fig. 11 obtained with the CIAO program is equivalent to the one that can be computed by using $x^{\prime}$ to define the $f_{r}$ cost function. Starting from the $n_{0}^{\prime}$ source node and the connector $\left(n_{0}^{\prime}, n_{1}^{\prime}\right)$ with cost $\langle 10,1\rangle$, the total cost of the tree $c_{n_{0}^{\prime}}$ is

$$
c_{n_{0}^{\prime}}=f_{r}\left(c_{n_{1}^{\prime}}\right)=\langle 10,1\rangle \times^{\prime} c_{n_{1}^{\prime}}
$$

Clearly, this framework can be used to solve the unicast problem as well, if the asked query include only one destination node, e.g. route $\left(n_{0},\left[n_{6}\right],[B, C]\right)$.

\subsection{Modality-based Steiner Tree Problems}

In this Section, as we provide in Sec. 4.3 for plain paths, we improve the tree search by including the possibility of considering some modalities associated with the use of the hyperarcs. Even in this case the justification is easy, since sometimes it may be useful to associate with each hyperarc also the information about the modality to be used to traverse that specific hyperarc. In this Section, we show an example using only two of the three modalities of Sec. 4.3: wired link with no encryption service $(w)$, and wireless link with no encryption service $(l)$. Other classes could collect slices of day time in which network links are preferred to be used (e.g. to

ACM Journal Name, Vol. V, No. N, 20YY. 


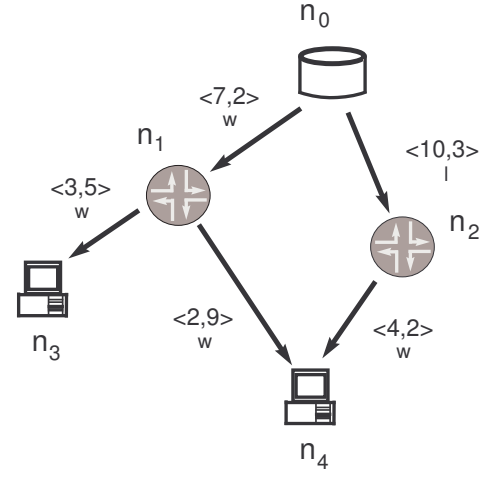

a)

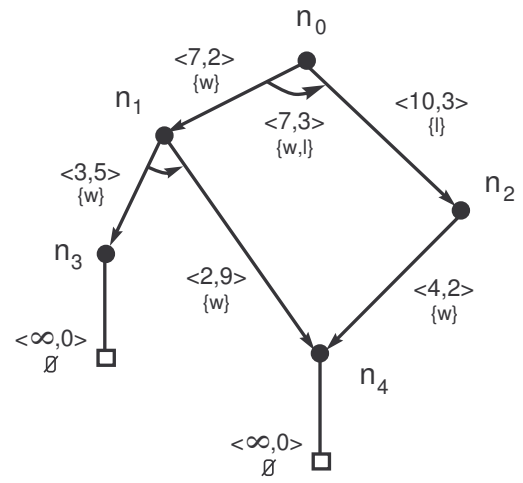

b)

Fig. 12. a) A network with modalities associated to the links, and b) the corresponding hypergraph.

better support the peaks of traffic), or label special conditions of use, e.g. to support "night back-up" or "black-out" events.

In Fig. 12 we show an example on how to pass from a network to a corresponding hypergraph with modalities (from Fig. $12 a$ to Fig. $12 b$ ): the modality associated with a connector is found by using the union operator (i.e. $U$ ) on the sets of modalities associated with each of the links represented by that connector. In the example of Fig. 12, 0-connectors have emptyset as label, since in this case we do not need any further information to finally reach a receiver; however, in general 0connector labels may contain the same modalities as the other $n$-connector labels, e.g. when they represent the internal structure of a sub network, as $\left(n_{9}\right)$ in Fig. $9 b$. For example, if the connection from $n_{0}$ to $n_{1}$ is wired, and the connection from $n_{0}$ to $n_{2}$ is wireless, the connector $\left(n_{0}, n_{1}, n_{2}\right)$ will be labelled with the $\{w, l\}$ modality set. Thus, edges are now represented in the following way:

$$
\text { edge(source_node, [dest_nodes], [bandwidth, cost], [list_of_modalities] })
$$

The query for the tree search must now be performed by including also the set of allowed modalities: if the set of modalities associated with a connector is a subset of the modalities asked in the query, then that connector can be used to build the tree. This can be practically accomplished by using, for example, the CIAO difference predicate between the two lists (sets) of modalities, or the sublist property.

For example (please refer to Fig. 12), asking for route $\left(n_{0},\left[n_{3}, n_{4}\right],[B, C],[w]\right)$ means that we are looking for paths made only with wired links (i.e. $w$ ). The $\left(n_{0}, n_{1}, n_{2}\right)$ connector cannot be used because its label is $\{w, l\}$ and we do not want to use wireless links (we remind that $l$ stands for wireless link with no encryption service). To include also that specific connector in the search, we have to ask the query route $\left(n_{0},\left[n_{3}, n_{4}\right],[B, C],[w, l]\right)$. Clearly, the final 0 -connectors are always included in trees because they have an emptyset label. 


\section{A LAST REFINEMENT ON SEMIRINGS FOR PARTIALLY-ORDERED PROB- LEMS}

As seen in Sec. 4.2 and 5.1, the costs on the connectors can be represented by vectors of costs, representing the QoS metric values of the network links. However, since we can have a partial order, two such pairs may possibly be incomparable, and this may lead to a strange situation while computing the semantics of a given goal. Considering the example in Sec. 4.2 and the related program in Table III, if we want to compute the cost and delay of the best path from $p$ to $v$, by giving the query :- p., the answer in this case is the value $\langle 7,7\rangle$. While the semiring value obtained in totally ordered SCLP programs represents the cost of one of the shortest paths, here it is possible that there are no routes with this cost: the obtained semiring value is in fact the greatest lower bound (w.r.t. both cost and delay) of the costs of all the paths from $p$ to $v$. This behavior comes from the fact that, if different refutations for the same goal have different semiring values, the SCLP framework combines them via the + operator of the semiring (which, in the case of our example, is the $\mathrm{min}^{\prime}$ operator of Sec. 4.2). If the semiring is partially ordered, it may be that $a+b$ is different from both $a$ and $b$. On the contrary, if we have a total order $a+b$ is always either $a$ or $b$.

This problem of course is not satisfactory, because usually one does not want to find the greatest lower bound of the costs of all paths from the given node to the destination node, but rather prefers to have one of the non-dominated paths. To solve this problem, we can add variables to the SCLP program, as we did in the previous Section, and also change the semiring. In fact, we now need a semiring which allows us to associate with the source node the set of the costs of all non-dominated path from there to the destination node. In other words, starting from the semiring $S=\langle A,+, \times, \mathbf{0}, \mathbf{1}\rangle$ (which, we recall, in the example of Sec. 4.2 is $\left\langle\mathbb{N}^{2}\right.$, min $\left.\left.^{\prime},+^{\prime},\langle+\infty,+\infty\rangle,\langle 0,0\rangle\right\rangle\right)$, we now have to work with the semiring $P^{H}(S)=\left\langle P^{H}(A), \uplus, \times^{*}, \emptyset, A\right\rangle$, where:

$-P^{H}(A)$ is the Hoare Power Domain [Smyth 1978] of $A$, that is, $P^{H}(A)=\{S \subseteq$ $A \mid x \in S, y \leq_{S} x$ implies $\left.y \in S\right\}$. In words, $P^{H}(A)$ is the set of all subsets of $A$ which are downward closed under the ordering $\leq_{S}$. It is easy to show that such sets are isomorphic to those containing just the non-dominated values. Thus in the following we will use this more compact representation for efficiency purposes. In this compact representation, each element of $P^{H}(A)$ will represent the costs of all non-dominated paths from a node to the destination node;

- the top element of the semiring is the set $A$ (its compact form is $\{\mathbf{1}\}$, which in our example is $\{\langle 0,0\rangle\})$;

- the bottom element is the empty set;

- the additive operation $\uplus$ is the formal union [Smyth 1978] that takes two sets and obtains their union;

- the multiplicative operation $x^{*}$ takes two sets and produces another set obtained by multiplying (using the multiplicative operation $\times$ of the original semiring, in our case +') each element of the first set with each element of the second one;

- the partial order of this semiring is as follows: $a \leq_{P^{H}(S)} b$ iff $a \uplus b=b$, that is for each element of $a$, there is an element in $b$ which dominates it (in the partial 
order $\leq_{S}$ of the original semiring).

From the theoretical results in [Smyth 1978], adapted to consider c-semirings, we can prove that $P^{H}(S)$ and its more compact form are indeed isomorphic. Moreover, we can also prove that given a c-semiring $S$, the structure $P^{H}(S)$ is a c-semiring as well [Bistarelli et al. 2002].

Theorem 6.1. Given a c-semiring $S=\langle A,+, \times, \mathbf{0}, \mathbf{1}\rangle$, the structure $P^{H}(S)=$ $\left\langle P^{H}(A), \uplus, \times^{*}, \emptyset, A\right\rangle$ obtained using the Power domain of Hoare operator is a csemiring.

Proof. The proof easily follows from the properties of the $\times$ operator in the csemiring $S$ and from the properties (commutativity, associativity, and idempotence) of the formal union $\uplus$ in $P^{H}(S)$.

Note that in this theorem we do not need any assumption over the c-semiring $S$. Thus the construction of $P^{H}(S)$ can be done for any c-semiring $S$. Notice also that, if $S$ is totally ordered, the c-semiring $P^{H}(S)$ does not give any additional information w.r.t. $S$. In fact, if we consider as a single element element the empty set (with the meaning that there are no paths) and the set containing only the bottom of $A$ (with the meaning that there exists a path whose cost is $\infty$ ), it is possible to build an isomorphism between $S$ and $P^{H}(S)$ by mapping each element $p$ (a set) of $P^{H}(A)$ onto the element $a$ of $A$ such that $a \in p$ and $a$ dominates all elements in the set $p$.

The only change we need to make to the program with variables, in order to work with this new semiring, is that costs now have to be represented as singleton sets. For example, clause $c_{p q}:-<2,4>$. will become $c_{p q}:-\{<2,4>\}$.

Still considering the example in Sec. 4.2, Let us now see what happens in our example if we move to this new semiring. First we give a goal like :- $\mathrm{p}(\mathrm{X})$. As the answer, we get a set of pairs, representing the costs of all non-dominated paths from $p$ to $v$. All these costs are non-comparable in the partial order, thus the user is requested to make a choice. However, this choice could identify a single cost or also a set of them. In this second case, it means that the user does not want to commit to a single path from the beginning and rather prefers to maintain some alternatives. The choice of one cost of a specific non-dominated path will thus be delayed until later. Other considerations on this semiring are given in [Bistarelli et al. 2002].

Most classical methods to handle multi-criteria SP problems find the shortest paths by considering each criteria separately, while our method deals with all criteria at once. This allows to obtain optimal solutions which are not generated by looking at each single criteria. In fact, some optimal solutions could be non-optimal in each of the single criteria, but still are incomparable in the overall ordering. Thus we offer the user a greater set of non-comparable optimal solutions. For example, by using a cost-delay multi-criteria scenario, the optimal solution w.r.t. cost could be $10 €$ (with a delay of $100 \mathrm{msec}$ ), while the optimal solution w.r.t. delay could be $10 \mathrm{msec}$ (with a cost of $100 €$ ). By considering both criteria together, we could also obtain the solution with 20 euro and $20 \mathrm{msec}$ !

Note that the considerations on partially-ordered problems of this Section clearly state for the multicast tree example in Sec. 5.1 as well. In this case, $P^{H}(S)=$ 
$\left\langle P^{H}(A), \uplus, \times^{*}, \emptyset, A\right\rangle$ uses the semiring for bandwidth-delay multi criteria: $S_{\text {Network }}=$ $\left\langle\langle\mathcal{B} \cup\{0,+\infty\}, \mathbb{N}\rangle,+^{\prime}, \times^{\prime},\langle 0,+\infty\rangle,\langle+\infty, 0\rangle\right\rangle$, where $\mathcal{B}$ is the set of bandwidth values, $+^{\prime}$ is $\langle\max , \min \rangle$ and $x^{\prime}$ is $\langle\min ,+\rangle$. Therefore, $x^{*}$ uses $\langle\min ,+\rangle\left(x^{\prime}\right)$ to compose two sets, and $\uplus$ the ordering $\leq_{S}$ defined by $\langle\max$, min $\rangle\left(+^{\prime}\right)$.

Finally, this method is applicable not only to the multi-criteria case, but to any partial order, giving us a general way to find a non-dominated path in a partiallyordered SP problem. It is important to notice here the flexibility of the semiring approach, which allows us to use the same syntax and computational engine, but on a different semiring, to compute different objects.

\subsection{Limiting the Number of Partially Ordered Solutions}

As presented in Sec. 6, we can use the Hoare Power Domain operator to retrieve the set of all the non-dominated paths (unicast) or trees (multicast) when we suppose that our network links have multiple and incomparable costs (e.g. bandwidth, cost and delay). This set of solutions is called the Pareto frontier and is guaranteed to contain all optimal solutions: all the solutions in this set are equivalently feasible. In other words, the Pareto frontier exactly captures the available trade-offs between the different QoS objectives. However, the use of partially ordered structures leads to the generation of a potentially exponential number of undominated solutions. When several of different paths/trees exist between the source and the receiver(s), it is therefore crucial to keep the number of configurations as low as possible through some form of approximation. However, the Hoare Power Domain operator can still be applied in case the sets of QoS costs have few elements and we really do not know how to refine the search, or if we know that few routes exist among nodes (these hypotheses limit the number of solutions).

We do not want to completely deviate from the incomparability property of the QoS metrics by adopting a total order, otherwise all the costs could be rephrased as a single one for each link, making the problem much more easier (e.g. the unicast problem can be solved in polynomial time [Cormen et al. 1990]) and less interesting, as explained in Sec. 3.

The proposed solution consists in avoiding a pointwise comparison of the single orderings representing the different criteria for the + operation of the semiring: we instead adopt a function that composes all the criteria in a single one and then chooses the best tuple of costs according to a total ordering. Each of the QoS criteria is composed by using a different importance value (i.e. a weight $w_{i}$ ). Theorem 6.2 proves that such function is still a valid + semiring operation for an Ordered Cartesian product of Weighted semirings [Bistarelli 2004; Bistarelli et al. $1997 b]$, i.e. $\left\langle\mathbb{R}^{+}, \min , \hat{+},+\infty, 0\right\rangle$ (where $\hat{+}$ is the arithmetic sum):

TheOREM 6.2. Given two Weighted semirings $S_{1}$ and $S_{2}$ and a relative preference for their element sets, i.e. $w_{1}, w_{2} \in \mathbb{R}^{+}$, we define the Ordered Cartesian product of $S_{1}$ and $S_{2} \equiv S_{f}=\left\langle\left\langle\mathbb{R}^{+} \times \mathbb{R}^{+}\right\rangle, f,\langle\hat{+}, \hat{+}\rangle,\langle+\infty,+\infty\rangle,\langle 0,0\rangle\right\rangle$. Given $\left\langle a_{1}, b_{1}\right\rangle,\left\langle a_{2}, b_{2}\right\rangle \in\left\langle\mathbb{R}^{+} \times \mathbb{R}^{+}\right\rangle, f$ (i.e. the + of the semiring) is defined as:

$$
f\left(\left\langle a_{1}, b_{1}\right\rangle,\left\langle a_{2}, b_{2}\right\rangle\right)= \begin{cases}\left\langle a_{1}, b_{1}\right\rangle & \text { if } w_{1} a_{1} \hat{+} w_{2} b_{1}>w_{1} a_{2} \hat{+} w_{2} b_{2} \\ \left\langle\min \left(a_{1}, a_{2}\right), \min \left(b_{1}, b_{2}\right)\right\rangle & \text { if } w_{1} a_{1} \hat{+} w_{2} b_{1}=w_{1} a_{2} \hat{+} w_{2} b_{2} \\ \left\langle a_{2}, b_{2}\right\rangle & \text { if } w_{1} a_{1} \hat{+} w_{2} b_{1}<w_{1} a_{2} \hat{+} w_{2} b_{2}\end{cases}
$$

ACM Journal Name, Vol. V, No. N, 20 YY. 
Then $S_{f}$ is a c-semiring.

Proof. Since the only change w.r.t. a classical Cartesian product of Weighted semirings is the + operator of $S_{f}$, we only need to check the properties of + given in Sec. 2. The function $f$ is commutative, associative, closed, idempotent, $\langle+\infty,+\infty\rangle$ is its unit element and $\langle 0,0\rangle$ its absorbing element: these properties easily follows from the properties of $\min$ and arithmetic sum and multiplication, which describe the $f$ expression. We only prove that $\times$ still distributes over $+\left(a_{i}, b_{i}, w_{i} \in \mathbb{R}^{+}\right)$:

$$
\begin{gathered}
\left\langle a_{1}, b_{1}\right\rangle \times\left(\left\langle a_{2}, b_{2}\right\rangle+\left\langle a_{3}, b_{3}\right\rangle\right)= \begin{cases}\left\langle\left(a_{1} \hat{+} a_{2}\right),\left(b_{1} \hat{+} b_{2}\right)\right\rangle \\
\left\langle\left( a_{1} \hat{+} \min \left(a_{2}, a_{3}\right),\left(b_{1} \hat{+} \min \left(b_{2}, b_{3}\right)\right\rangle\right.\right. & \text { if } \text { cond }_{1} \\
\left\langle\left(a_{1} \hat{+} a_{3}\right),\left(b_{1} \hat{+} b_{3}\right)\right\rangle & \text { if } \text { cond }_{2}\end{cases} \\
\quad\left(\left\langle a_{1}, b_{1}\right\rangle \times\left\langle a_{2}, b_{2}\right\rangle\right)+\left(\left\langle a_{1}, b_{1}\right\rangle \times\left\langle a_{3}, b_{3}\right\rangle\right)= \\
\begin{cases}\left\langle\left(a_{1} \hat{+} a_{2}\right),\left(b_{1} \hat{+} b_{2}\right)\right\rangle & \text { if } \operatorname{cond}_{4} \\
\left\langle\min \left(a_{1} \hat{+} a_{2}, a_{1} \hat{+} a_{3}\right), \min \left(b_{1} \hat{+} b_{2}, b_{1} \hat{+} b_{3}\right)\right\rangle & \text { if } \operatorname{cond}_{5} \\
\left\langle\left(a_{1} \hat{+} a_{3}\right),\left(b_{1} \hat{+} b_{3}\right)\right\rangle & \text { if } \operatorname{cond}_{5}\end{cases}
\end{gathered}
$$

Where $\operatorname{cond}_{1}$ is $w_{1} a_{2} \hat{+} w_{2} b_{2}>w_{1} a_{3} \hat{+} w_{2} b_{3}$ and $\operatorname{cond}_{4}$ is $w_{1}\left(a_{1} \hat{+} a_{2}\right) \hat{+} w_{2}\left(b_{1} \hat{+} b_{2}\right)>$ $w_{1}\left(a_{1} \hat{+} a_{3}\right) \hat{+} w_{2}\left(b_{1} \hat{+} b_{3}\right)$; by simplifying both sides of $\operatorname{cond}_{4}$ we obtain that cond $_{1} \equiv$ $\operatorname{cond}_{2}$. In the same way we can prove that $\operatorname{cond}_{2} \equiv \operatorname{cond}_{5}$ if and $\operatorname{cond}_{3} \equiv \operatorname{cond}_{6}$. Therefore, $\times$ distributes over + .

Notice that the proof can be easily extended for an Ordered Cartesian product of $n>2$ Weighted semirings. Notice also that we can assemble an Ordered Cartesian product even for $n$ Probabilistic semirings [Bistarelli 2004; Bistarelli et al. 1997b], and even for semirings in general, as claimed in Theo. 6.3:

TheOREM 6.3. We consider two identical semirings $S_{1}, S_{2}=\langle A,+, \times, \mathbf{0}, \mathbf{1}\rangle$ where $\times$ is cancellative [Bistarelli and Gadducci 2006]. We can define an Ordered Cartesian product $S_{f}$ as $\langle\langle A \times A\rangle, f,\langle\times, \times\rangle,\langle\mathbf{0}, \mathbf{0}\rangle,\langle\mathbf{1}, \mathbf{1}\rangle\rangle$, where $f$ is defined as:

$$
f\left(\left\langle a_{1}, b_{1}\right\rangle,\left\langle a_{2}, b_{2}\right\rangle\right)= \begin{cases}\left\langle a_{1}, b_{1}\right\rangle & \text { if } a_{1} \times b_{1}>_{S_{1,2}} a_{2} \times b_{2} \\ \left\langle a_{1}+a_{2}, b_{1}+b_{2}\right\rangle & \text { if } a_{1} \times b_{1}=S_{S_{1,2}} a_{2} \times b_{2} \\ \left\langle a_{2}, b_{2}\right\rangle & \text { if } a_{1} \times b_{1}<_{S_{1,2}} a_{2} \times b_{2}\end{cases}
$$

Then $S_{f}$ is a c-semiring.

Proof. Notice that we use the same + and $\times$ operators of $S_{1}, S_{2}$ also in the definition of $f$, thus their properties still hold. For this reason, we can easily prove that the + (as defined by $f$ ) of the semiring is commutative, associative, closed, idempotent, $\langle\mathbf{0}, \mathbf{0}\rangle$ is its unit element and $\langle\mathbf{1}, \mathbf{1}\rangle$ its absorbing element. The cancellative property is needed to prove the that $\times$ distributes over + :

$$
\left\langle a_{1}, b_{1}\right\rangle \times\left(\left\langle a_{2}, b_{2}\right\rangle+\left\langle a_{3}, b_{3}\right\rangle\right)= \begin{cases}\left\langle\left(a_{1} \times a_{2}\right),\left(b_{1} \times b_{2}\right)\right\rangle & \text { if } \text { cond }_{1} \\ \left\langle\left(a_{1} \times\left(a_{2}+a_{3}\right),\left(b_{1} \times\left(b_{2}+b_{3}\right)\right)\right\rangle\right. & \text { if } \text { cond }_{2} \\ \left\langle\left(a_{1} \times a_{3}\right),\left(b_{1} \times b_{3}\right)\right\rangle & \text { if } \text { cond }_{3}\end{cases}
$$




$$
\begin{gathered}
\left(\left\langle a_{1}, b_{1}\right\rangle \times\left\langle a_{2}, b_{2}\right\rangle\right)+\left(\left\langle a_{1}, b_{1}\right\rangle \times\left\langle a_{3}, b_{3}\right\rangle\right)= \\
\begin{cases}\left\langle\left(a_{1} \times a_{2}\right),\left(b_{1} \times b_{2}\right)\right\rangle & \text { if } \text { cond }_{4} \\
\left\langle\left(a_{1} \times a_{2}\right)+\left(a_{1} \times a_{3}\right),\left(b_{1} \times b_{2}\right)+\left(b_{1} \times b_{3}\right)\right\rangle & \text { if } \text { cond }_{5} \\
\left\langle\left(a_{1} \times a_{3}\right),\left(b_{1} \times b_{3}\right)\right\rangle & \text { if } \text { cond }_{6}\end{cases}
\end{gathered}
$$

Where cond $_{1}$ is $a_{2} \times b_{2}>_{S_{1,2}} a_{3} \times b_{3}$ and cond $_{4}$ is $\left(a_{1} \times a_{2}\right) \times\left(b_{1} \times b_{2}\right)>_{S_{1,2}}$ $\left(a_{1} \times a_{3}\right) \times\left(b_{1} \times b_{3}\right)$. Since $\times$ is cancellative, we can simplify both sides of cond $_{4}$ and we obtain that $\operatorname{cond}_{1} \equiv \operatorname{cond}_{4}$. In the same way we can prove that $\operatorname{cond}_{2} \equiv$ cond $_{5}$ if and cond $_{3} \equiv$ cond $_{6}$. Therefore, $\times$ distributes over + .

With Theo. 6.2 and Theo. 6.3 we show that multiple semirings of the same type (e.g. Weighted or Probabilistic) can be composed together according to some expressed preferences. In this way, the resulting tuples are totally ordered and the final solution consists in the most preferred one. Ad-hoc compositions can be used also to merge different semirings in a single one, e.g. Weighted and Probabilistic. However, according to the definition of $f$ in Theo. 6.3 (similar considerations hold for Theo. 6.2), $f\left(\left\langle a_{1}, b_{1}\right\rangle,\left\langle a_{2}, b_{2}\right\rangle\right)=\left\langle a_{1}+a_{2}, b_{1}+b_{2}\right\rangle$ if $a_{1} \times b_{1}=_{S_{1,2}} a_{2} \times b_{2}$, and thus $f$ returns the lowest upper bound of the two couples. As already said in Sec. 6 , this result does not represent a "real" solution. Nonetheless, this problem can be overcome by collecting all the best equivalent couples in the same set, i.e. applying the Hoare Power Domain operator (see Sec. 6).

Corollary 6.1. Given an Ordered Cartesian product $S_{f}=\langle\langle A \times A\rangle, f,\langle\times, \times\rangle$, $\langle\mathbf{0}, \mathbf{0}\rangle,\langle\mathbf{1}, \mathbf{1}\rangle\rangle$ as described in Theo. 6.2 and the Hoare Power Domain operator $P^{H}$, then $P^{H}\left(S_{f}\right)$ is a semiring.

Proof. Given the results in Theo. 6.1 (see Sec. 6), we can easily assemble the Hoare Power Domain over the semiring proposed in Theo. 6.3, by using the Hoare Power Domain operator (see Sec. 6).

A similar result can be proved for the semiring assembled with Theo. 6.2 (i.e. for the Weighted semirings), by applying to it the Hoare Power Domain operator as well.

\section{SOLVING THE PROBLEM IN PRACTICE}

\subsection{Scale-free Networks}

Small-world networks may belong to three classes: single-scale, broad-scale, or scale-free depending on their connectivity distribution $P(k)$, which is the probability that a randomly selected node has exactly $k$ edges. Scale-free networks follow a power law of the generic form $P(k) \backsim k^{-\gamma}$ [Faloutsos et al. 1999]: in words, in these networks some nodes act as "highly connected hubs" (with a high degree), although most nodes are of low degree. Intuitively, the nodes that already have many links are more likely to acquire even more links when new nodes join in the graph: this is the so-called "rich gets richer" phenomenon. These hubs are the responsible for the small world phenomenon. The consequences of this behavior are that, compared to a random graph with the same size and the same average degree, the average path

ACM Journal Name, Vol. V, No. N, 20 YY. 
length of the scale-free model is somewhat smaller, and the clustering coefficient of the network is higher, suggesting that the graph is partitioned in sub-communities.

Several works as [Faloutsos et al. 1999; Vazquez et al. 2002] show that Internet topology can be modeled with scale-free graphs: in [Vazquez et al. 2002] the authors distinguish between the Autonomous System (AS) level, where each AS refers to one single administrative domain of the Internet, and the Internet Router level (IR). At the IR level, we have graphs with nodes representing the routers and links representing the physical connections among them; at the AS level graphs each node represents an AS and each link represents a peer connection trough the use of the Border Gateway Protocol (BGP) protocol. Each AS groups a generally large number of routers, and therefore the AS maps are in some sense a coarse-grained view of the IR maps. The same authors of [Vazquez et al. 2002] confirm the scalefree property for both these kinds of graphs with a $\gamma=2.1 \pm 0.1$, even if IR graphs have a power-law behavior smoothed by an exponential cut-off: for large $k$ the connectivity distribution follows a faster decay, i.e. we have much less nodes with a high degree. This truncation is probably due to the limited number of physical router interfaces. In [Cohen and Havlin 2003] the authors prove that scale free networks with $2<\gamma<3$ have a very small diameter, i.e. $\ln \ln N$, where $N$ is the number of nodes in the graph.

Therefore, we decided to test our QoS routing framework on this kind of networks because they properly model both the AS and the IR levels.

\subsection{Implementing the Framework}

To develop and test a practical implementation of our model, we adopt the Java Universal Network/Graph Framework (JUNG) [O'Madadhain et al. 2003], a software library for the modeling, analysis, and visualization of a graph or network. With this library it is also possible to generate scale-free networks according to the preferential attachment proposed in [Barabasi and Albert 1999]: each time a new vertex $v_{n}$ is added to the network $G$, the probability $p$ of creating an edge between an existing vertex $v$ and $v_{n}$ is $p=(\operatorname{degree}(v)+1) /(|E|+|V|)$, where $|E|$ and $|V|$ are respectively the current number of edges and vertices in $G$. Therefore, vertices with higher degree have a higher probability of being selected for attachment. We generated the scale-free network in Fig. 13 (the edges are undirected) and then we automatically produced the corresponding program in CIAO (where the edges are directed), as shown in Sec. 5.2. The reported statistics suggest the scale-free nature of our network: a quite high clustering coefficient, a low average shortest path and a high variability of vertex degrees (between average and max). These features are evidences of the presence of few big hubs that can be used to shortly reach the destinations.

However, with the CIAO program representing the network in Fig. 13, all the queries we tried to perform over that graph were explicitly stopped after 5 minutes without discovering the best QoS route solution. Therefore, a practical implementation definitely needs a strong performance improvement: in Sec. 8 we show some possible solutions that could all be used also together. In Sec. 8.3 we show an implementation of the exactly same program in ECLiPSe [Apt and Wallace 2007]: in addition, we use branch-and-bound to prune the search and we claim that only this technique is sufficient to experience a feasible response time for the queries. 


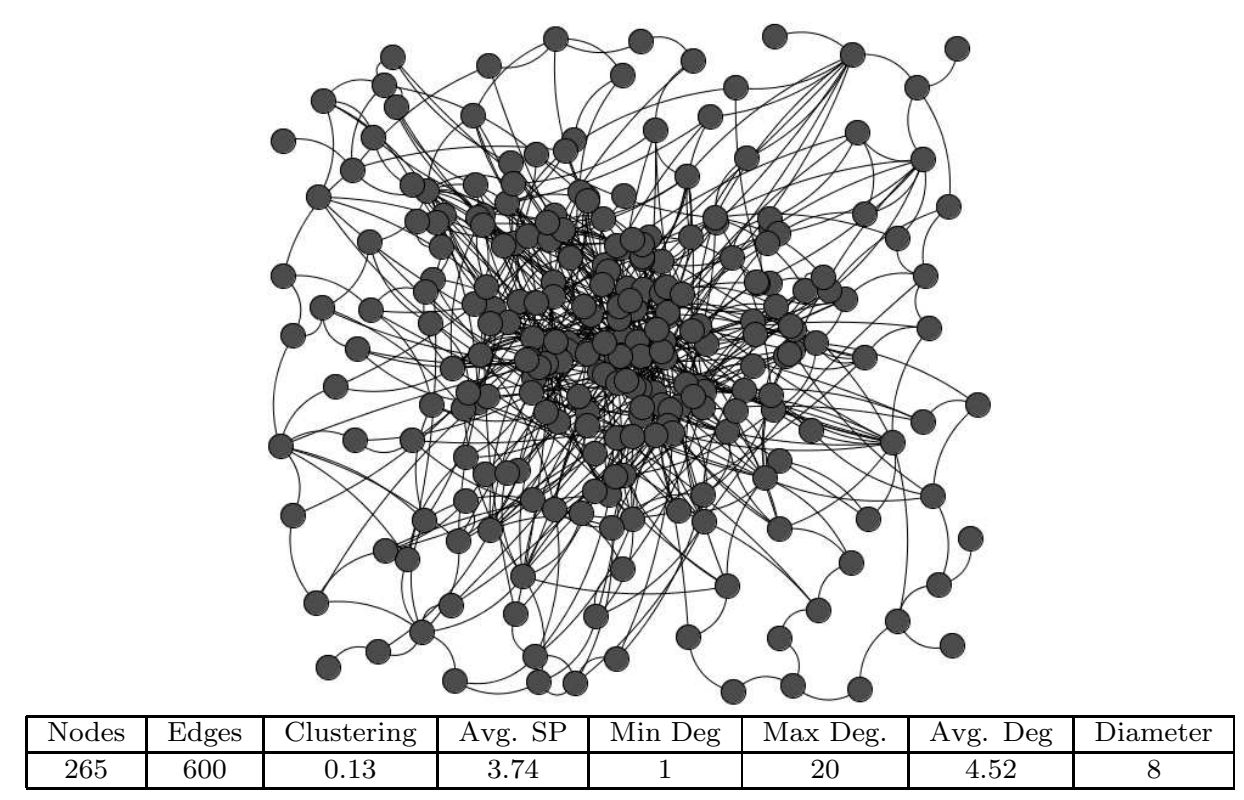

Fig. 13. The test scale-free network and the related statistics.

\section{PERFORMANCE AND FEASIBLE ENCODINGS}

Although the framework we present in this paper is conceived as a declarative and expressive mean to represent the QoS routing problem, some of the used encodings represent an obstacle towards a real use on practical cases. Our study is clearly not aimed at a successful performance comparison with dedicated algorithms running inside routers or network devices: we instead desire to model many different routing constraints (e.g. routing and policy constraints) all inside the same framework. The power of this model is in the facility with which routing constraints and network bounds in general can be expressed and added to pre-existing rules. However, we need also a feasible implementation to obtain and check a solution for real-case networks, even if not performing as well as the algorithms reported in Sec. 3.2 and Sec. 3.3. All these works are focused only on some metrics (e.g. DVMA [Rouskas and Baldine 1997] considers only delay and jitter) or adopts ad-hoc heuristics to relax the problem. Since in Sec. 7.2 we prove that a straightforward implementation is not feasible in practice, in this Section we provide the methods to lighten these encodings and tackle down the performance problems. For the reason that we use a general and open framework, we will suggest general strategies to enhance the results. However, we think that more specific techniques can be used as well.

Notice that the techniques we are going to present but not directly implement in practice (as tabling in Sec. 8.2) for sake of brevity, have however a strong and accepted background concerning their efficiency.

\subsection{Using a cut function to reduce the number of solutions}

In Sec. 6.1 we solve the (potentially) exponential space problem linked to the Pareto optimal frontier of the multicriteria solutions: in that case, we "flat" the partial

ACM Journal Name, Vol. V, No. N, 20YY. 
ordering by composing all the criteria together and using a total ordering on the result.

However, reducing all the QoS costs to a single one is a coarse simplification that can be applied only in some cases: it is not always possible to completely rank all the preferences among themselves (e.g. the "user" could not have clear ideas, or it could not be possible to "mix" different metrics together), and it could be often pleasing to show more results to the final user. Moreover, as reported in literature, with a single metric the problem becomes much less interesting: for example, the unicast problem becomes solvable in polynomial time, instead of to be NP-Complete (see Sec. 3.1).

For this reason, in Def. 8.1 we define a cut function that can be applied each time on the result of the formal union (i.e. $\uplus)$ of the Hoare Power Domain defined in Sec. 6. After the cut, the set contains only the best tuples of costs, chosen with the criteria defined by the function. Definition 8.1 is presented for the Weighted semirings, but other ad-hoc cuts can be defined for other types of semirings, just in case the criteria wanted to reduce the number of solutions cannot be represented with a semiring-based structure (as in Theo. 6.2). In words, the costs of a tuple $t$ are composed in a single cost $c_{t}$ with the aid of a weight for each tuple element: this weight can change in a predefined interval, thus different $c_{t}$ can be obtained. Then, $t$ is deleted from the set if, for each of its $c_{t}$, there always exists another tuple $v$ in the set and a cost $c_{v}>c_{t}$.

DEFINITION 8.1. We consider a set $P$ of partially ordered $n$-tuples $\left\langle a_{1}, a_{2}, \ldots, a_{n}\right\rangle$, where $a_{i} \in \mathbb{R}^{+}$in $\left\langle\mathbb{R}^{+}\right.$, min, $\left.\hat{+},+\infty, 0\right\rangle$ (i.e. a Weighted semiring); each $a_{i}$ is associated with a weight $w_{i}$ in the interval $\left[k_{i}-\epsilon_{i}, k_{i}+\epsilon_{i}\right]$ and $k_{i}, \epsilon_{i} \in \mathbb{R}^{+}$. A cut function can be defined as cut $(P)=P_{\text {cut }} \subseteq P$, where $P_{\text {cut }}=\left\{\left\langle b_{1}, b_{2}, \ldots, b_{n}\right\rangle \in\right.$ $P \mid \nexists\left\langle c_{1}, c_{2}, \ldots, c_{n}\right\rangle \in P .\left(w_{1} b_{1} \hat{+} w_{2} b_{2} \hat{+} \ldots \hat{+} w_{n} b_{n}\right)<\left(w_{1} c_{1} \hat{+} w_{2} c_{2} \hat{+} \ldots \hat{+} w_{n} c_{n}\right), \forall w_{i} \in$ $\left.\left[k_{i}-\epsilon_{i}, k_{i}\right], i \in\{1 . . n\}\right\}$

Therefore, we reduce the number of solutions and we continue considering a partial order and not a total one (which is important for us, as explained before), but we discard "bad" tuples of cost, where "bad" is according to the expressed preferences. Notice that not all the different criteria must have an associated weight, and the cut can be performed only considering a subset of metrics.

Notice also that this cut function can be easily modelled with CIAO Prolog clauses by considering the solutions as lists and by using the delete predicate on the elements that do not satisfy the given conditions. Notice also that the preference criteria are different from the ones described in Theo. 6.2: i.e. it can be proved that the final set of solution obtained with the Hoare Power Domain operator (see Theo. 6.1) is a subset of the set found with the cut function in Def. 8.1.

\subsection{Tabled Soft Constraint Logic Programming and Network Decomposition}

In logic programming, the basic idea behind tabling (or memoing) is that the calls to tabled predicates are stored in a searchable structure together with their proven instances: subsequent identical calls can use the stored answers without repeating the computation. This collection of tabled subgoals paired with their answers, generally referred to as call table and answer table respectively, is consulted whenever a new call, $C$, to a tabled predicate is issued. If $C$ is similar to a tabled subgoal 
$S$, then the set of answers, $A$, associated with $S$ may be used to satisfy $C$. In such instances, $C$ is resolved against the answers in $A$, and hence we refer to $C$ as a consumer of $A$ (or $S$ ). If there is no such $S$, then $C$ is entered into the call table and is resolved against program clauses. As each answer is derived during this process, it is inserted into the answer table entry associated with $C$ if it contains information not already in $A$. Furthermore, left recursion need not lead to non-termination because identical subgoals are not evaluated, and thus the possible infinite loops are avoided.

Tabling improves the computability power of Prolog systems and for this reason many programming frameworks have been extended in this direction. Due to the power of this extension, many efforts have been made to include it also in CLP, thus leading to the Tabled Constraint Logic Programming (TCLP) framework. In [Cui and Warren 2000] the authors present a TCLP framework for constraint solvers written using attributed variables; however, when programming with attributed variables, the user have to take care of of many implementation issues such as constraint store representation and scheduling strategies. A more recent work [Schrijvers and Warren 2004] explains how to port Constraint Handling Rules (CHR) to XSB (acronym of eXtended Stony Brook), and in particular its focus is on technical issues related to the integration of CHR with tabled resolution: as a result, a CHR library is presently combined with tabling techniques within the XSB system. CHR is a high-level natural formalism to specify constraint solvers and propagation algorithms. This a further promising framework where to solve QoS routing problems and improve the performance (for example, tabling efficiency is shown in [Ramakrishnan et al. 1995]), since soft constraints have already been successfully ported to the CHR system [Bistarelli et al. 2002]. Hence, part of the soft constraint solving can be performed once and reused many times.

One more consideration that can be taken into account while trying to reduce the complexity, is that large networks, as Internet, are already partitioned into different Autonomous System (AS) [Moy 1998], or however, into subnetworks. An AS is a collection of networks and routers under the control of one entity (or sometimes more) that presents a common routing policy to the Internet. AS can be classified by observing the types of traffic traversing them. A multihomed AS maintains connections to more than one other AS; however, it would not allow traffic from one AS to pass through on its way to another AS. A stub AS is only connected to a single AS. A transit AS provides connections through itself to the networks connected to it. Considering Fig. 14, network $A S 1$ can use the transit AS3 to connect to network $A S 2$. An $A S$ number (or ASN) uniquely identifies each AS on the internet (i.e. $A S 1, A S 2$ and $A S 3$ ).

As shown in Fig. 14, in each AS (or subnetwork in general) we can find a table with the QoS routing goals concerning the destinations (routers and hosts) within its bounds, by using tabling techniques. At this point, these tables helps to find the routes that span multiple ASs and the search procedure is considerably speeded up: the routes internal to each AS can be composed together by simply using the links connecting the border routers. For example, consider when a sender in $A S 1$ needs to start a multicast communication towards some receivers in $A S 2$ and $A S 3$ : the routers inside $A S 1$ can use Table 1 to find the routes from the source to the border

ACM Journal Name, Vol. V, No. N, 20 YY. 


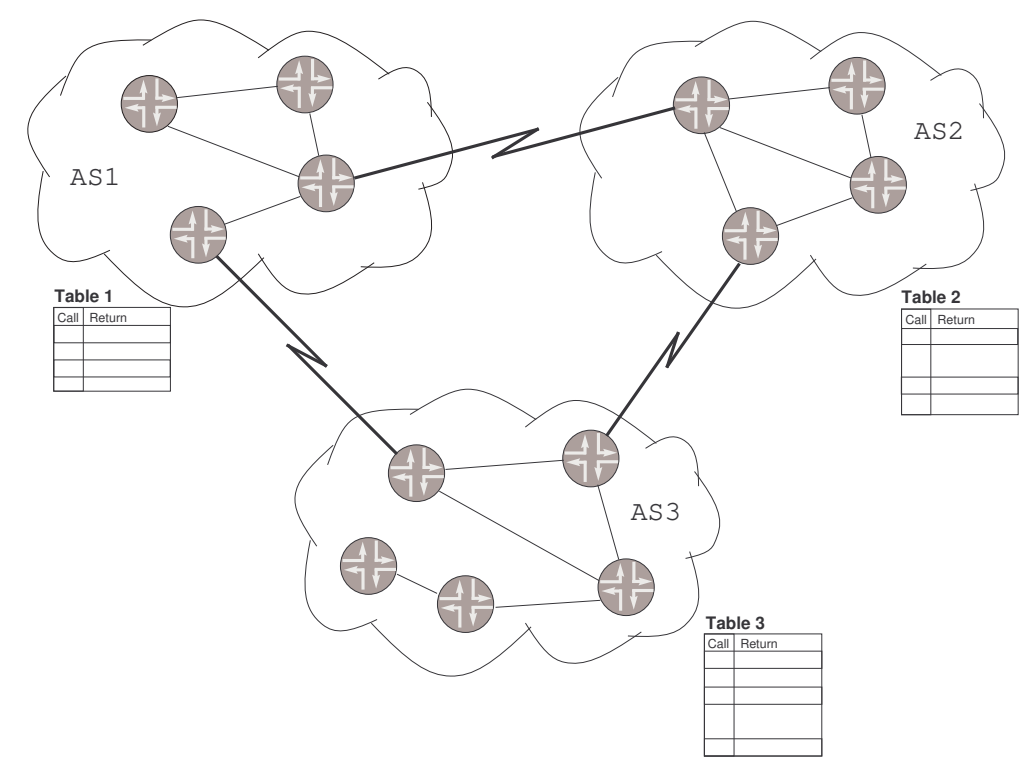

Fig. 14. A network subdivided in Autonomous Systems; each AS can store in its border routers a table with the goals related to that specific AS.

routers of $A S 1$ (i.e. it can communicate with other ASs). Then, the border routers in AS2 and AS3 respectively use Table 2 and Table 3 to find the second and final part of the route towards the receivers inside their AS. The procedure of finding such a goal table for a single AS is much less time consuming than finding it for the whole not-partitioned network. Clearly, the fundamental premise to obtain a substantial benefit from this technique is to have strongly-connected subnetworks and few "bridges" among them.

\subsection{An implementation in ECLiPSe}

As shown in Sec. 5.1, the representation of the f-star of node in the multicast model can be composed by a total of $O\left(2^{n}\right)$ connectors, thus in the worst case it is exponential in the number of graph nodes. This drawback, which is vigorously perceived in strongly connected networks, and together with considering a real case network linking hundreds of nodes, would heavily impact on the time-response performance during a practical application of our model. Therefore, it is necessary to elaborate some improvements to reduce the complexity of the tree search, for example by visiting as few branches of the SCLP tree as possible (thus, restricting the solution space to be explored). For this reason, we provide a further implementation by using the ECLiPSe [Apt and Wallace 2007] system.

ECLiPSe is a software system for the development and deployment of constraint programming applications, e.g. in the areas of planning, scheduling, resource allocation, timetabling, transport and more. It contains several constraint solver libraries, a high-level modelling and control language, interfaces to third-party solvers, an integrated development environment and interfaces for embedding into 


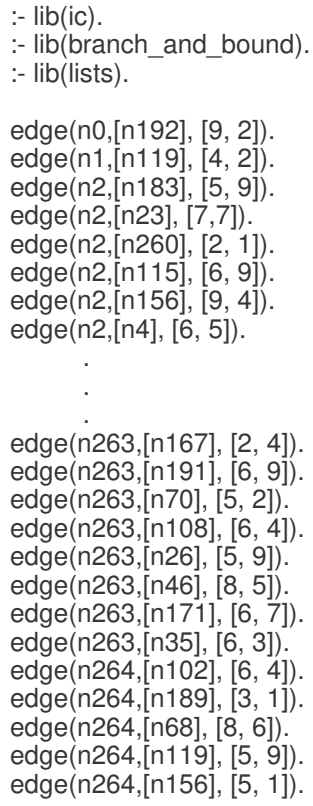

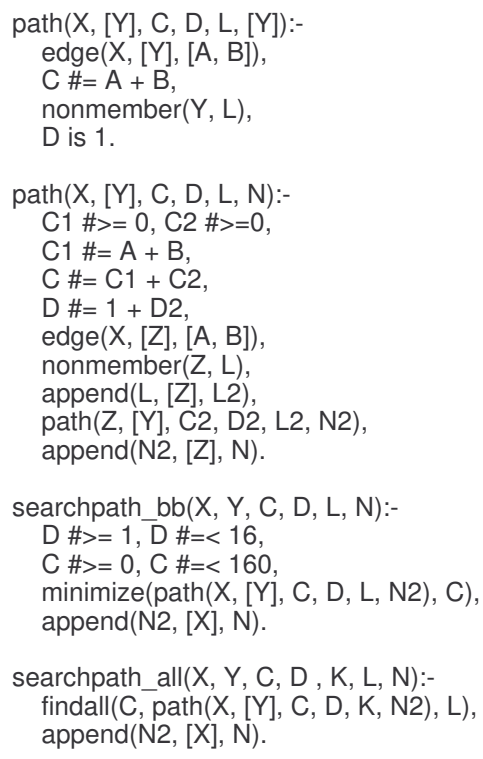

Fig. 15. The representation in ECLiPSe (with branch-and-bound optimization) of the QoS routing problem for the network in Fig. 13; clearly, only some of the 600 edges are shown.

host environments [Apt and Wallace 2007]. We decided to use ECLiPSe because of its extendibility and efficiency due to its wide range of optimization libraries (e.g. on symmetry breaking). In particular, we exploit the branch_and_bound library in order to reduce the space of explored solutions and consequently improve the performance. Branch-and-bound is a well-known technique for optimization problems, which is used to immediately cut away not promising partial solutions, by basing on a "cost" function.Unfortunately, as far as we know, ECLiPSe does not support tabling techniques (introduced in 8.2) and therefore it cannot be adopted to compose the benefits of both techniques.

In Fig. 15 we show a program in ECLiPSe that represents the unicast QoS routing problem for the scale-free network in Fig. 13. We decided to show only the unicast case for sakes of clarity, but feasible time responses can be similarly obtained for the multicast case (i.e. searching for a tree instead of a plain path) by working on the branch-and-bound interval of explored costs, as we will better explain in the following. Clearly, in Fig. 15 we report only some of the 600 edges of the network.

The code in Fig. 15 has been automatically generated with a Java program using JUNG, as done for the CIAO program in Sec. 7.2: the corresponding text file is 30Kbyte. The size can be halved by not printing the reverse links and generating them with a specific clause, if each link and its reverse one have the same cost.

The branch-and-bound optimization is achieved with minimize $(+$ Goal, ?Cost) (importing the branch_and_bound library) in the searchpath_bb clause in Fig. 15, where the Goal is a nondeterministic search routine (the clauses that describe the 


\begin{tabular}{|c|c|c|c|}
\hline \multicolumn{2}{|c|}{ ECLiPSe 5.10 Toplevel } & - & \\
\hline \multicolumn{4}{|c|}{ File Query Iools Help } \\
\hline \multicolumn{4}{|c|}{ Query Entry } \\
\hline leclipse & \#: $:$ searchpath_bb $(\mathrm{n} 2, \mathrm{n} 262, \mathrm{C}, \mathrm{D}$, & $[\mathrm{n} 2], \mathrm{N}) . \pm$ & \pm \\
\hline run & make & interrupt & \\
\hline \multicolumn{4}{|c|}{ Results } \\
\hline \multicolumn{4}{|c|}{ 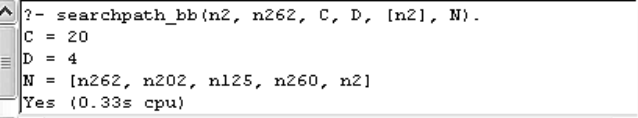 } \\
\hline \multicolumn{4}{|l|}{$v<1$} \\
\hline \multicolumn{4}{|c|}{ Output and Error Messages } \\
\hline \begin{tabular}{|l} 
Found \\
Found \\
Found \\
Found \\
Found \\
Found \\
Found \\
Found \\
Found \\
Found \\
Found \\
Found \\
Found \\
Found \\
Found \\
Found \\
Found \\
Found \\
Found \\
Found \\
Found \\
Found \\
Found \\
Found
\end{tabular} & $\begin{array}{l}\text { a solution with cost } 144 \\
\text { a solution with cost } 130 \\
\text { a solution with cost } 122 \\
\text { a solution with cost } 115 \\
\text { a solution with cost } 111 \\
\text { a solution with cost } 108 \\
\text { a solution with cost } 107 \\
\text { a solution with cost } 87 \\
\text { a solution with cost } 73 \\
\text { a solution with cost } 70 \\
\text { a solution with cost } 59 \\
\text { a solution with cost } 56 \\
\text { a solution with cost } 54 \\
\text { a solution with cost } 47 \\
\text { a solution with cost } 46 \\
\text { a solution with cost } 44 \\
\text { a solution with cost } 40 \\
\text { a solution with cost } 39 \\
\text { a solution with cost } 31 \\
\text { a solution with cost } 28 \\
\text { a solution with cost } 25 \\
\text { a solution with cost } 22 \\
\text { a solution with cost } 20 \\
\text { no solution with cost } 1.0 . .19 .0\end{array}$ & & \\
\hline$<$ & & & 3 \\
\hline
\end{tabular}

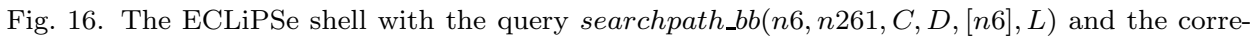
sponding found result for the program in Fig. 15.

path structure) that instantiates a Cost variable (i.e. the QoS cost of the path) when a solution is found. Notice that for each of the edges of the network we randomly generated two different QoS costs by using the java.util.Random Class, each of them in the interval [1..10]. Therefore the cost of link is represented by a couple of values. In order to model the semiring we propose in Theo. 6.2, the cost of the path is computed by summing the two QoS features together (i.e. $A$ and $B$ in Fig. 15): we compute $w_{1} A+w_{2} B$ and we suppose $w_{1}=w_{2}=1$, i.e. the composed cost of a link is in the interval [2..20]. ECLiPSe natively allows to apply a branch-and-bound procedure focused only on a single cost variable, but ad-hoc techniques can be developed to consider also the cut function presented in Def. 8.1, in order to keep a real multicriteria preference for the QoS features.

The two clauses searchpath_bb and searchpath_all represent the queries that can be asked to the system: they respectively use and not use the branch-and-bound optimization, i.e. searchpath_all finds all the possible paths in order to find the best one. In order to describe the structure of a searchpath_bb query (see Fig. 15), we take as example searchpath_bb $(n 2, n 262, C, D,[n 2], L)$ : with this query we want to find the best path between the nodes $n 2$ and $n 262, C$ is the cost of the path (used also by the branch-and-bound pruning), $D$ is the number of hops, $L$ (in Fig. 15) is the list of already traversed nodes and $N$ is a list used to collect the nodes of the 


\begin{tabular}{|c|c|}
\hline $\begin{array}{l}D \#>=1, \\
D \#=<16\end{array}$ & $\begin{array}{l}\text { These two constraints are used to limit the depth (i.e. the number } \\
\text { of hops) of the path we want to find. For the example in Fig. } 15 \text { it } \\
\text { was computed as Diameter } \times 2=8 \times 2=16 \text {. It is a good } \\
\text { overestimation since we are dealing with a scale-free network } \\
\text { (see Sec } 7.1 \text { ). }\end{array}$ \\
\hline$D \#=1+D 2$ & Used to compute the depth of the path. \\
\hline $\begin{array}{c}\mathrm{C} 1 \#>=0, \mathrm{C} 2 \#>=0 \\
\mathrm{C} 1 \#=A+B \\
C \#=C 1+C 2\end{array}$ & $\begin{array}{l}\text { Four constraints are used to compute the cost of the path: it is } \\
\text { the cost of an edge (i.e. } C 1 \text { is obtained by summing the two QoS } \\
\text { features } A \text { and } B \text { ) plus the cost of the remaining part of the path } \\
\text { (i.e. } C 2 \text { ). Clearly, both } C 1 \text { and } C 2 \text { must be greater than } 0 \text {. }\end{array}$ \\
\hline $\begin{array}{c}C \#>=0, \\
C \#=<160\end{array}$ & $\begin{array}{l}\text { Used to limit the space of cost values: its reduction sensibly } \\
\text { improves the performance. It is possible to start the search with a } \\
\text { small threshold and then raise it if no solution is found. For the } \\
\text { example in Fig. } 15 \text { it was computed as the maximum possible } \\
\text { cost of a path: EdgeMaxCost } x \text { Diameter }=20 \times 8=160 \text {. }\end{array}$ \\
\hline
\end{tabular}

Table VII. The description of the constraints used in Fig. 15.

path (in reverse order). The result of this query is reported in Fig. 16, by showing directly the ECLiPSe window: the best cost value (i.e. 20) was found after 0.33 seconds with a path of 4 hops, i.e. $n 2-n 260-n 125-n 202-n 262$.

The corresponding query searchpath_all $(n 6, n 261, C, D, K,[n 6], N)(K$ is the list of solutions found by the findall predicate), which does not use the branch-andbound pruning (and constraints), was explicitly interrupted after 10 minutes without finding the goal. Other queries are satisfied in even less than one second, depending on the efficiency of the pruning efficiency for the specific case.

To better describe and accelerate the search we added also some constraints, which are explained in Tab. 8.3. In Fig. 15 we also import the hybrid integer/real interval arithmetic constraint solver of ECLiPSe to use them, i.e. the ic library. Notice that the constraints depending on the Diameter of the network (i.e. 8, as shown in Fig. 13) limit the search space and provides a mild approximation at the same time: in scale-free networks, the average distance between two nodes can be $\ln \ln N$, where $N$ is the number of nodes [Cohen and Havlin 2003] (see also Sec. 7.1). Therefore, considering a max depth of the path as twice the diameter value (i.e. 16) still results in a large number of alternative routes, since, for the scale-free network in Fig. 13, this value is 4-5 times the average shortest path of the network (i.e. 3.74 as shown in Fig. 13).

In order to show the scalability property of our framework, in Tab. VIII we summarize the performance results of 50 queries executed on three distinct scalefree networks with a different number of nodes: $n=50, n=265$ (i.e. the network in Fig. 13) and $n=877$. These statistics are related to the Min/Max/Average Time needed to obtain a path, its Average Cost and its Max/Average Depth. For each query, the source and destination nodes have been randomly generated. We can see that Max Time sensibly differs from the Average Time, and this is due to the poor efficiency of the branch-and-bound pruning in some cases. However, this technique performs very well in most of cases, as the low Average Time Tab. VIII shows (even for $n=877$ ).

ACM Journal Name, Vol. V, No. N, $20 Y$ Y. 


\begin{tabular}{|c|c|c|c|c|c|c|}
\hline Nodes & Min Time & Max Time & Avg. Time & Avg. Cost & Avg. Depth & Max Depth \\
\hline 50 & $\sim 0 \mathrm{~s}$ & $0.45 \mathrm{~s}$ & $0.1 \mathrm{~s}$ & 17.54 & 3.04 & 7 \\
\hline 265 & $0.02 \mathrm{~s}$ & $77.12 \mathrm{~s}$ & $4.08 \mathrm{~s}$ & 29.8 & 5.46 & 11 \\
\hline 877 & $0.5 \mathrm{~s}$ & $40.05 \mathrm{~s}$ & $4.89 \mathrm{~s}$ & 37.72 & 6.72 & 14 \\
\hline
\end{tabular}

Table VIII. Some performance statistics obtained with the ECLiPSe framework (with branch-andbound), collected on three different size networks (i.e. 50, 265 and 1000 nodes). On each network we performed 50 queries.

Comparable performance results are achievable as well also for the multicast case, by enforcing the structure of the tree with other ad-hoc constraints: for example, by constraining the width of the searched tree to the number of the multicast receivers in the query, since it is useless to find wider trees. Moreover, the problem can be first over-constrained and then relaxed step-by-step if no solution is found. For example, we can start by searching a solution in the cost interval [0..35] and then, if the best solution is not included in this interval, setting the interval to [36..70] (and so on until the best solution is found). Notice that in this way we strongly speed-up the search while preserving all the information, due to the characteristics of the branchand-bound technique. This behaviour can be easily reproduced in ECLiPSe, since the customizable options of bb_min (+Goal,?Cost,?Options) (i.e. another clause to express branch-and-bound) include the [From..To] interval parameters.

At last, we are confident that the ECLiPSe system can be used to further improve the performance, since it is possible to change the parameters of branch-and-bound, e.g. by changing the strategy after finding a solution [Apt and Wallace 2007]: continue search with the newly found bound imposed on Cost, restart or perform a dichotomic after finding a solution, by splitting the remaining cost range and restart search to find a solution in the lower sub-range. If it fails, the procedure assumes the upper sub-range as the remaining cost range and splits again. Moreover, it is possible to add Local Search to the tree search, and to program specific heuristics [Apt and Wallace 2007].

8.3.1 Further reducing the dimension of $n$-connectors. One more enhancement that can be accomplished to reduce the size of a node's neighborhood (w.r.t. the given query) for the multicast distribution, is the inclusions of program facts that describe the topology of the network (or part of it). In this way, like in classic network routing, we can immediately remove from the search the not involved clusters or those clusters we do not want to cross for policy reasons. For example, we can add the list of reachable ASs directly in each connector: if a connector allows us to reach $\left\{A S_{1}, A S_{2}, A S_{4}\right\}$ but not $\left\{A S_{3}, A S_{5}\right\}$, we can use the first list as the additional routing information linked to that connector $\left(A_{i}\right.$ represent constant names). If the intersection between the ASs related to the receivers in the query and the list of a given connector is empty, then we can avoid considering that connector in the search since it will reach only not interesting nodes. An graphical example of this behaviour is given in Fig. 17.

Clearly, other hierarchical partitions can be adopted instead of large ASs: for example we can consider simple subnetworks if we have to deal with a small departmental networks. Considering scale-free networks (see Sec. 7.1), these improvements are strongly needed for hub nodes, i.e. the backbone nodes of the network 


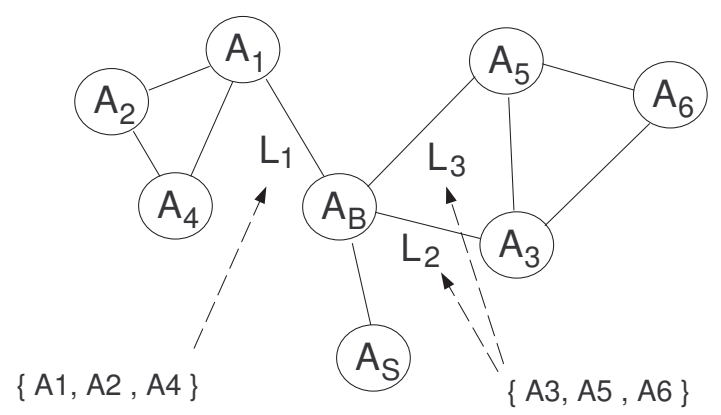

Fig. 17. Routing information can be added to the link clauses to avoid parts of the network (e.g. the $L_{1}$ link can be avoided if the destination is $A_{6}$ ).

with a high degree: these nodes connect a lot of separate networks together and thus we can avoid to explore those branched not touched by the query. This kind of relaxation can be easily programmed in CIAO Prolog, with lists of terms (to represent the list of ASs reached by a 1-connector), union predicate (to join the lists of 1-connectors) and difference predicate to check that the AS lists of the query and the obtained $n$-connector have a non-empty intersection (otherwise that connector is useless for the proposed query).

\section{CONCLUSIONS}

We have described a method to represent and solve the unicast/multicast QoS routing problem with the combination of graph/hypergraph and SCLP programming: i) the best path found in this way corresponds to the best unicast route distributing (for example) multimedia content from the source to the only receiver; ii) the same considerations are also valid for the best tree found over an and-or graph, since it corresponds to the best multicast distribution tree towards all the receivers. The best path/tree optimizes objectives regarding QoS performance, e.g. minimizing the global bandwidth consumption or reducing the delay, and can satisfy constraints on these metric values at the same time. The structure of a $c$-semiring defines the algebraic framework to model the costs of the links, and the SCLP framework describes and solves the SCSP problem in a declarative fashion. Since several distinct criteria must be all optimized (the costs of the arcs may include multiple QoS metric values), the best route problem belongs to the multi-criteria problem class, i.e. it can result in a partially-ordered problem. Moreover we have seen also how to deal with modality-based problems, relating them to preferences connected to policy routing rules. Therefore, the model proposed in this paper can be used to reason upon (and solve!) CBR, that is, in general, a NP-Complete problem.

In the future, we plan to enrich this framework by using Soft Concurrent Constraint Programming (SCCP) [Bistarelli et al. 2006] to handle the interactions among the routing devices and the receivers, and, consequently, we would like to introduce new "soft" operations (e.g. a retract of a constraint) to enable the release of the resources reserved by the receivers of the communication, introducing a non-monotonic evolution of the constraint store (which is not allowed in classical

ACM Journal Name, Vol. V, No. N, 20 YY. 
SCCP). A second step should consist in further expanding the SCCP framework with some simple primitives which allows to specify timing constraints [Bistarelli et al. 2007]. Time critical aspects are essential to the management of QoS, and, in general, when modelling the possible interactions among distributed or concurrent systems. These entities must continuously react to the inputs coming from the environment and act in an appropriate manner.

A further extension to our QoS framework could be the introduction of probabilistic metrics as the weight of the graph-links: we could consider this value as the probability of packet loss on that connection, or the probability of a connection existence between two nodes in the network. In this case, the global probability of existence of a path between two nodes $p$ and $v$ depends on the probability of all the possible different paths connecting $p$ and $v$ in the graph. The problem is represented by the composition of the different probabilities of these paths, which cannot be easily modelled with a c-semiring, but we could use the formulation given for semiring valuation and introduced in Sec. 2 , where the + operator of the c-semiring is non-idempotent.

At last, we will study if it is possible to represent the operators used in MST and ST algorithms (see Sec. 3.1) with semiring structures.

\section{REFERENCES}

Apt, K. R. And Wallace, M. 2007. Constraint Logic Programming using Eclipse. Cambridge University Press, New York, NY, USA.

Barabasi, A. L. AND Albert, R. 1999. Emergence of scaling in random networks. Science 286, 509.

Berman, L., Kou, L., and Markowsky, G. 1979. A fast algorithm for steiner trees. Acta Informatica 15, 2 (December), 141-145.

Bistarelli, S. 2004. Semirings for Soft Constraint Solving and Programming. Lecture Notes in Computer Science, vol. 2962. Springer, London, UK.

Bistarelli, S., Frühwirth, T., And Marte, M. 2002. Soft constraint propagation and solving in chrs. In SAC '02: Proceedings of the 2002 ACM symposium on Applied computing. ACM Press, New York, NY, USA, 1-5.

Bistarelli, S., Gabrielli, M., Meo, M. C., and Santini, F. 2007. Timed concurrent constraint programs. In Doctoral Program Informal Proceedings, CP'0\%.

Bistarelli, S. And GadducCI, F. 2006. Enhancing constraints manipulation in semiring-based formalisms. In European Conference on Artificial Intelligence (ECAI). 63-67.

Bistarelli, S., Montanari, U., And Rossi, F. 1995. Constraint Solving over Semirings. In Proc. IJCAI95. Morgan Kaufman, 624-630.

Bistarelli, S., Montanari, U., And Rossi, F. 1997a. Semiring-based Constraint Logic Programming. In Proc. IJCAI97. Morgan Kaufman, 352-357.

Bistarelli, S., Montanari, U., And Rossi, F. 2002. Soft constraint logic programming and generalized shortest path problems. Journal of Heuristics 8, 1, 25-41.

Bistarelli, S., Montanari, U., and Rossi, F. 2006. Soft concurrent constraint programming. ACM Trans. Comput. Logic 7, 3, 563-589.

Bistarelli, S., Montanari, U., And Rossi, F. March 1997b. Semiring-based Constraint Solving and Optimization. Journal of the ACM 44, 2, 201-236.

Bistarelli, S., Montanari, U., Rossi, F., And Santini, F. 2007. Modelling multicast qos routing by using best-tree search in and-or graphs and soft constraint logic programming. Electr. Notes Theor. Comput. Sci. 190, 3, 111-127.

Bueno, F., Cabeza, D., Carro, M., Hermenegildo, M., Lopez, P., and Puebla, G. 1997. The ciao prolog system. reference manual. The CIAO System Documentation Series-TR CLIP3/97.1. 
Chen, S. And NAhrstedt, K. 1998. An overview of quality of service routing for next-generation high-speed networks: Problems and solutions. IEEE Network 12, 6 (November/December), 64-79.

Chen, S., Nahrstedt, K., And Shavitt, Y. 2000. A QoS-aware multicast routing protocol. In INFOCOM (3). 1594-1603.

Cohen, R. And Havlin, S. 2003. Scale-free networks are ultrasmall. Phys. Rev. Lett. 90, 5 (Feb), 058701.

Cormen, T. T., Leiserson, C. E., And Rivest, R. L. 1990. Introduction to algorithms. Mit Press, Cambridge, MA, USA.

Crawley, E., Nair, R., Rajagopalan, B., And Sandick, H. 1998. RFC 2386: A framework for QoS-based routing in the Internet. Informational.

Cui, B. And Warren, D. S. 2000. A system for tabled constraint logic programming. In $C L$ ' 00 : Proceedings of the First International Conference on Computational Logic. Springer-Verlag, London, UK, 478-492.

de Nicola, R., Ferrari, G. L., Montanari, U., Pugliese, R., and Tuosto, E. 2003. A formal basis for reasoning on programmable qos. In Verification: Theory and Practice, N. Dershowitz, Ed. Lecture Notes in Computer Science, vol. 2772. Springer, 436-479.

Faloutsos, M., Faloutsos, P., And Faloutsos, C. 1999. On power-law relationships of the internet topology. In SIGCOMM '99. ACM Press, 251-262.

Garey, M. R. And Johnson, D. S. 1979. Computers and Intractability: A Guide to the Theory of NP-Completeness. W. H. Freeman \& Co., New York, NY, USA.

Georget, Y. And Codognet, P. 1998. Compiling semiring-based constraints with clp (fd, s). In $C P$ '98: International Conference on Principles and Practice of Constraint Programming. Springer-Verlag, London, UK, 205-219.

Hirsch, D. AND Tuosto, E. 2005. Shreq: Coordinating application level qos. In SEFM '05: Proceedings of the Third IEEE International Conference on Software Engineering and Formal Methods. IEEE Computer Society, Washington, DC, USA, 425-434.

JAFFar, J. AND MAher, M. J. 1994. Constraint logic programming: A survey. Journal of Logic Programming 19/20, 503-581.

Kompella, K. And Awduche, D. 2001. Notes on path computation in constraint-based routing. Internet Draft.

Korkmaz, T. And Krunz, M. 2001. Multi-constrained optimal path selection. In INFOCOM. 834-843.

Kuipers, F. A., Korkmaz, T., Krunz, M., and Mieghem, P. V. 2004. Performance evaluation of constraint-based path selection algorithms. IEEE Network 18, 5, 16-23.

Loo, B. T., Hellerstein, J. M., Stoica, I., and Ramakrishnan, R. 2005. Declarative routing: extensible routing with declarative queries. In SIGCOMM '05: Proceedings of the 2005 conference on Applications, technologies, architectures, and protocols for computer communications. ACM, New York, NY, USA, 289-300.

Ma, Q. And Steenkiste, P. 1997. Quality of service routing for traffic with performance guarantees.

MAmmeRI, Z. 2004. Towards a formal model for qos specification and handling in networks. In IWQOS. IEEE, 148-152.

Martelli, A. And Montanari, U. 1978. Optimizing decision trees through heuristically guided search. Commun. ACM 21, 12, 1025-1039.

Mieghem, P. V., Neve, H. D., And Kuipers, F. A. 2001. Hop-by-hop quality of service routing. Computer Networks 37, 3/4, 407-423.

Mohri, M. 2002. Semiring frameworks and algorithms for shortest-distance problems. J. Autom. Lang. Comb. 7, 3, 321-350.

MoY, J. 1998. RFC 2328: OSPF version 2. Standard.

O'Madadhain, J., Fisher, D., White, S., And Boey, Y. 2003. The JUnG (Java Universal Network/Graph) framework. Tech. rep., UC Irvine.

ACM Journal Name, Vol. V, No. N, 20YY. 
Paul, P. And Raghavan, S. V. 2002. Survey of qos routing. In ICCC '02: Proceedings of the 15th international conference on Computer communication. International Council for Computer Communication, Washington, DC, USA, 50-75.

Ramakrishnan, I. V., RaO, P., Sagonas, K. F., Swift, T., And Warren, D. S. 1995. Efficient tabling mechanisms for logic programs. In International Conference on Logic Programming. The MIT Press, 697-711.

Régin, J.-C., Petit, T., Bessière, C., And Puget, J.-F. 2000. An original constraint based approach for solving over constrained problems. In $C P^{\prime}$ '02: Proceedings of the 6th International Conference on Principles and Practice of Constraint Programming. Springer-Verlag, London, UK, 543-548.

Rosen, E., Viswanathan, A., And Callon, R. 2001. Multiprotocol Label Switching Architecture.

Rouskas, G. N. AND BALDine, I. 1997. Multicast routing with end-to-end delay and delay variation constraints. IEEE Journal of Selected Areas in Communications 15, 3, 346-356.

SchriJvers, T. AND Warren, D. S. 2004. Constraint handling rules and tabled execution. In $I C L P$, B. Demoen and V. Lifschitz, Eds. Lecture Notes in Computer Science, vol. 3132. Springer, $120-136$.

Sмyтн, M. B. 1978. Power domains. Journal of Computer and System Sciences 16, 1 (Feb.), $23-36$.

TARJAn, R. E. 1979. A unified approach to path problems. Tech. rep., Stanford, CA, USA.

Vazquez, A., Pastor-Satorras, R., and Vespignani, A. 2002. Internet topology at the router and autonomous system level.

WANG, B. AND HoU, J. 2000. Multicast routing and its QoS extension: problems, algorithms, and protocols. IEEE Network 14.

WANG, Z. 1999. On the complexity of quality of service routing. Inf. Process. Lett. 69, 3, 111-114.

WANG, Z. AND CROWCROFT, J. 1996. Quality-of-service routing for supporting multimedia applications. IEEE Journal on Selected Areas in Communications 14, 7, 1228-1234.

WILSON, N. 2004. Bounds and pre-processing for local computation of semiring valuations.

Winter, P. 1987. Steiner problem in networks: a survey. Netw. 17, 2, 129-167.

XiaO, X. And Ni, L. M. 1999. Internet qos: A big picture. IEEE Network 13, 2 (March), 8-18.

Younis, O. And FAhmy, S. 2003. Constraint-based routing in the internet: Basic principles and recent research. IEEE Communications Surveys and Tutorials 5, 1, 2-13. 